\title{
Effect of circular holes on the ratchet limit and crack tip plastic strain range in a centre cracked plate
}

\author{
Haofeng Chen*, Weihang Chen, Tianbai Li, James Ure \\ Department of Mechanical Engineering, University of Strathclyde, Glasgow, G1 1XJ, UK
}

\begin{abstract}
In this paper a centre cracked plate subjected to cyclic tensile loading and cyclic bending moment is considered. The effect of circular holes drilled in the region of the crack tip on the ratchet limit and crack tip plastic strain range is studied. Direct evaluation of the ratchet limit and crack tip plastic strain range is solved by employing the new Linear Matching Method (LMM). Parametric studies involving hole diameter and location are investigated. The optimum hole location for reducing the crack tip plastic strain range with the least reduction in ratchet limit is identified, and located at a distance $10 \%$ of the semi-crack length from the crack tip on the side opposite the ligament for both cyclic tensile loading and cyclic bending moment cases. It is also observed that the optimum location is independent of the hole size for both cyclic loading cases.
\end{abstract}

Keywords: ratchet limit, shakedown, linear matching method, centre cracked plate

\section{Introduction}

Cracks, which develop during manufacturing or the service period of structures, affect the load capacity, residual strength, life and integrity of the structure. These cracks may grow and cause material, economical and human damages therefore it is necessary to increase the residual strength and service life of the cracked structures by arresting the crack growth. Several methods have been employed to arrest crack growth, such as external adhesive patching across the crack used in aircraft industry [1-2], the method of pressing steel balls and drilling holes in front of the crack tip so that when the crack approaches the hole it will become blunted and be arrested. The method of drilling stop holes is well known to reduce the stress intensity factor and studies have been carried out in this area [3]. However, the effects of the location and diameter of circular holes on the ratchet limit and crack tip plastic strain range, which provides information concerning fatigue crack growth in a low cycle fatigue assessment, have not been undertaken.

\footnotetext{
* Corresponding author.

Email: haofeng.chen@strath.ac.uk

Tel. +44 $1415482036 \quad$ Fax. +441415525105
} 


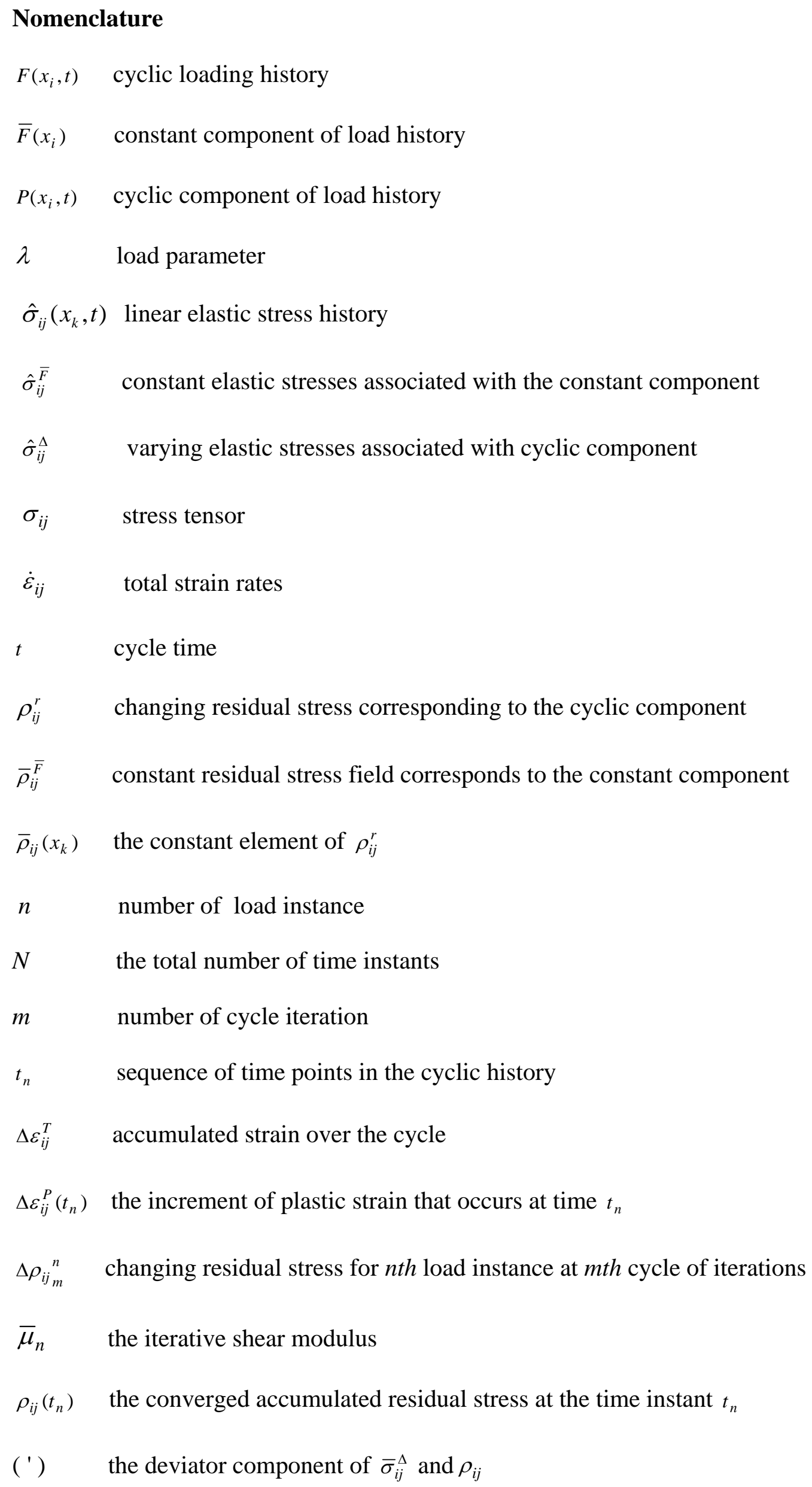




$$
\begin{array}{ll}
\varepsilon_{i j}^{c} & \text { kinematically admissible strain } \\
\sigma_{i j}^{c} & \text { state of associated stress with } \varepsilon_{i j}^{c} \text { at yield } \\
\sigma_{y} & \text { uniaxial yield stress of material } \\
a & \text { half crack length } \\
\frac{W}{a} & \text { the width ratio of the centre cracked plate }
\end{array}
$$

$\frac{L}{a} \quad$ the length ratio of the centre cracked plate

D diameter of the hole

$X, Y$ co-ordinate system located at the crack tip

$X_{0}, Y_{0}$ the coordinates of the hole's centre

$\bar{\sigma}_{p} \quad$ mean tension or constant uniaxial tension

$\Delta M \quad$ bending moment range

$\bar{\sigma}_{p o} \quad$ reference mean tensile loading or constant uniaxial tension

$\Delta M_{0}$ reference reversed bending moment range

$\lambda_{R} \quad$ ratchet limit multiplier from the case of a centre cracked plate with holes

$\lambda_{R 0} \quad$ ratchet limit multiplier from the case of a centre cracked plate without holes

$\Delta \varepsilon_{p} \quad$ maximum plastic strain range from the case of a centre cracked plate with holes

$\Delta \varepsilon_{p 0}$ maximum plastic strain range from the case of a centre cracked plate without holes 
In the analysis of structures subjected to cyclic loading histories for an elastic-perfectly plastic material, the component will either shakedown or ratchet. The elastic shakedown limit is the highest cyclic load under which a material shakes down to an elastic response after the first few load cycles. When the elastic shakedown limit is exceeded, the structure may experience either plastic shakedown or ratchetting. In many applications, it is too conservative for a structure to be within the elastic shakedown limit [4]. Plastic shakedown or alternating plasticity, under which a local low cycle fatigue failure mode occurs, may be permitted, provided that during its design life the effect of low cycle fatigue is taken into consideration. Ratchetting, which ultimately leads to incremental plastic collapse, must be avoided in any case, since it may lead to intolerable deformations. And for this reason it is desirable to calculate the ratchet limit of a structure under cyclic load condition. In addition, the evaluation of the ratchet limit is particularly useful for structures with stress raisers, such as cracks. In such structures, due to the presence of the elastic stress singularity at the crack tip the shakedown condition becomes invalid. Hence a finite shakedown limit does not exist anymore. However, the procedures for identifying the ratchet limit are still valid. This is due to the closed cycles of plastic strains occurring at the crack tip, enabling the evaluation of the finite ratchet limits. As a result, a method on the determination of the ratchet limit for cracked bodies is particularly desirable.

Many direct methods for modelling cyclic plasticity behaviour of the material have been developed in the past decades $[5,6,7,8,9,10]$. These direct methods use simple material models, i.e. elastic-perfectly plastic, and consider a load domain that contains all possible load paths between the extremes, thus eliminating the need to know the precise load path which is normally required by the detailed step-by-step analysis [11]. Among these direct methods, the Linear Matching Method (LMM) $[9,10]$ is recognized as one of the most powerful methods. The LMM is distinguished from other simplified methods by ensuring that equilibrium and compatibility are satisfied at each stage. In addition to the shakedown analysis method [12], the LMM has been extended beyond the range of most other direct methods by including the evaluation of ratchet limit and plastic strain range $[9,10,13]$ and high temperature material behaviour [14]. The new ratchet limit method [13] has been verified to be capable of evaluating the ratchet limit and plastic strain range for defect-free components subjected to cyclic load conditions involving multi-load extremes. However, the application of this latest ratchet limit method on cracked structures has not been undertaken. Thus it is particularly important to extend the ratchet analysis procedure so that the method can be adopted to investigate the behaviour of cracked structures subjected to cyclic load histories. 
The aim of the present paper is to employ a new simplified method for the evaluation of ratchet limit and plastic strain range under the LMM framework for cracked bodies subjected to cyclic load conditions, and to analyse the effect of the circular hole on the ratchet limit and crack tip plastic strain range in a centre cracked plate using the proposed method. In the present paper, a centre cracked plate with symmetric holes subjected to two load conditions, cyclic uniaxial loading and cyclic bending moment with constant tensile loading, is considered by assuming plane strain condition. The effect of circular holes on the ratchet limit and crack tip plastic strain range, which is considered to be a better similitude parameter than the stress intensity range for the fatigue crack growth behaviour [15] in a stable cycle, is presented. Parametric studies involving hole diameter and locations are carried out. The optimum location for reducing the crack tip plastic strain range with the least reduction in ratchet limit is identified considering the fact that reducing the plastic strain range will increase the component's fatigue life while an unchanged ratchet limit will keep the cyclic loading capacity of the cracked plate. An ABAQUS [11] step-by -step inelastic analysis is also carried out to verify the obtained ratchet limit by the proposed method.

\section{Numerical Procedures}

\subsection{Cyclic load history}

Let us consider the problem of an elastic-perfectly plastic body subjected to a general cyclic load history $F\left(x_{i}, t\right)$, which can be decomposed into cyclic component $P\left(x_{i}, t\right)$ and constant component $\lambda \bar{F}\left(x_{i}\right)$, i.e.

$$
F\left(x_{i}, t\right)=\lambda \bar{F}\left(x_{i}\right)+P\left(x_{i}, t\right)
$$

where $\lambda$ is a load parameter and the variation is considered over a typical cycle $0 \leq t \leq \Delta t$ in a cyclic state. The corresponding linear elastic stress history is denoted by $\hat{\sigma}_{i j}\left(x_{k}, t\right)$ as

$$
\hat{\sigma}_{i j}\left(x_{k}, t\right)=\lambda \hat{\sigma}_{i j}^{\bar{F}}\left(x_{k}\right)+\hat{\sigma}_{i j}^{\Delta}\left(x_{k}, t\right)
$$

where $\hat{\sigma}_{i j}^{\bar{F}}$ denotes the constant elastic stresses due to the constant component $\bar{F}\left(x_{i}\right)$ and $\hat{\sigma}_{i j}^{\Delta}$ denotes the varying elastic stresses due to the cyclic component $P\left(x_{i}, t\right)$.

\subsection{Asymptotic Cyclic Solution}

For the cyclic problem defined above, the stresses and strain rates will become asymptotic to a cyclic state where;

$$
\sigma_{i j}(t)=\sigma_{i j}(t+\Delta t) \quad \dot{\varepsilon}_{i j}(t)=\dot{\varepsilon}_{i j}(t+\Delta t)
$$


The cyclic stress solution may be expressed in terms of four components, the varying elastic stress solution corresponding to the cyclic component of the load history, the associated changing residual stress field, the constant elastic stress solution due to the constant component of the load history and its associated constant residual stress. Hence, the general form of the stress solution for the cyclic problems involving changing and constant residual stress fields is given by

$$
\sigma_{i j}\left(x_{k}, t\right)=\hat{\sigma}_{i j}^{\Delta}\left(x_{k}, t\right)+\rho_{i j}^{r}\left(x_{k}, t\right)+\lambda \hat{\sigma}_{i j}^{\bar{F}}\left(x_{k}\right)+\bar{\rho}_{i j}^{\bar{F}}\left(x_{k}\right)
$$

where $\bar{\rho}_{i j}^{\bar{F}}$ denotes a constant residual stress field in equilibrium with zero external load and corresponds to the constant component of the elastic stress history $\lambda \hat{\sigma}_{i j}^{\bar{F}}$. The $\rho_{i j}^{r}$ is the changing residual stress corresponding to the cyclic component of the elastic stress $\hat{\sigma}_{i j}^{\Delta}$ during the cycle and it satisfies the condition;

$$
\rho_{i j}^{r}\left(x_{k}, 0\right)=\rho_{i j}^{r}\left(x_{k}, \Delta t\right)=\bar{\rho}_{i j}\left(x_{k}\right)
$$

where $\bar{\rho}_{i j}\left(x_{k}\right)$ is the constant element of $\rho_{i j}^{r}$.

To evaluate the ratchet limit numerically for a component subjected to a predefined cyclic load history to withstand an extra constant load, we decouple the evaluation of the changing residual stress $\rho_{i j}^{r}(t)$ and the constant residual stress $\bar{\rho}_{i j}^{\bar{F}}$ so that the varying part and constant part of the residual stress may be evaluated separately. Hence, the whole numerical procedure includes two stages; The first stage is to calculate the history of the changing residual stress field $\rho_{i j}^{r}(t)$ associated with the predefined cyclic load history and the corresponding plastic strain ranges associated with a low cycle fatigue assessment. The second stage is to locate the ratchet limit by a conventional shakedown analysis where a constant residual stress $\bar{\rho}_{i j}^{\bar{F}}$ is evaluated and the elastic stress history is augmented by the changing residual stress calculated in the first stage.

\subsection{Numerical procedure for the varying residual stress field and plastic strain range}

The Linear Matching Method procedure for the assessment of residual stress history and the associated plastic strain range due to the cyclic component of the load history is described below in terms of $\mathrm{N}$ discrete time points. Following the same procedure as [14], for a strictly convex yield condition, the only instants when plastic strains can occur are at the vertices of the stress history $\hat{\sigma}_{i j}^{\Delta}\left(t_{n}\right), \mathrm{n}=1$ to $\mathrm{N}$, where $\mathrm{N}$ represents the total number of time instants, $t_{1}, t_{2} \ldots \ldots . t_{N}$, of the load extremes where plastic strain occurs and $t_{n}$ corresponds to a sequence of time points in the load history. Then the plastic strain accumulated during the cycle $\Delta \varepsilon_{i j}^{T}=\sum_{n=1}^{N} \Delta \varepsilon_{i j}^{P}\left(t_{n}\right)$ where $\Delta \varepsilon_{i j}^{P}\left(t_{n}\right)$ is the increment of plastic strain that occurs at time $t_{n}$. The entire iterative procedure includes a number 
of cycles, where each cycle contains $N$ iterations associated with $N$ load instances. The first iteration is to evaluate the changing residual stress $\Delta \rho_{i j}^{1}$ associated with the elastic solution $\hat{\sigma}_{i j}^{\Delta}\left(t_{1}\right)$ at the first load instance. Define $\Delta \rho_{i j_{m}}^{n}$ as the evaluated changing residual stress for $n t h$ load instance at $m t h$ cycle of iterations, where $n=1,2, \ldots \mathrm{N}$ and $m=1,2, \ldots \mathrm{M}$. At each iteration, the above changing residual stress $\Delta \rho_{i j}{ }_{m}^{n}$ for $n$th load instance at $m$ th cycle of iteration is calculated. When the convergence occurs at the mth cycle of iterations, the summation of changing residual stresses at $\mathrm{N}$ time points must approach to zero $\left(\sum_{n=1}^{N} \Delta \rho_{i j}{ }^{n}=0\right)$ due to the stable cyclic response. Hence the constant element of the residual stress for the cyclic loading history is

$$
\rho_{i j}^{r}(0)=\rho_{i j}^{r}(\Delta t)=\bar{\rho}_{i j}
$$

and determined by

$$
\bar{\rho}_{i j}=\sum_{n=1}^{N} \Delta \rho_{i j_{1}}{ }^{n}+\sum_{n=1}^{N} \Delta \rho_{i j_{2}}{ }^{n}+\cdots+\sum_{n=1}^{N} \Delta \rho_{i j}{ }_{M-1}^{n}
$$

The corresponding converged increment of plastic strain occurring at time $t_{n}$ is calculated by

$$
\Delta \varepsilon_{i j}^{p}\left(t_{n}\right)=\frac{1}{2 \bar{\mu}_{n}}\left[\bar{\sigma}_{i j}^{\Delta^{\prime}}\left(t_{n}\right)+\rho_{i j}{ }^{\prime}\left(t_{n}\right)\right]
$$

where $\bar{\mu}_{n}$ is the iterative shear modulus and notation ( ' ) refers to the deviator component of $\bar{\sigma}_{i j}^{\Delta}$ and $\rho_{i j} \cdot \rho_{i j}\left(t_{n}\right)$ is the converged accumulated residual stress at the time instant $t_{n}$, i.e.

$$
\rho_{i j}\left(t_{n}\right)=\bar{\rho}_{i j}+\sum_{k=1}^{n} \Delta \rho_{i j}{ }_{M}
$$

The detailed iterative procedure for the evaluation of the residual stress history and associated plastic strain range has been implemented into ABAQUS through user subroutines UMAT and given in [13].

\subsection{Numerical procedure for the ratchet limit}

Once the history of the accumulated residual stress field $\rho_{i j}\left(t_{n}\right)$ at the time instance $t_{n}$ associated with the cyclic component of the load history has been calculated, the numerical technique for the ratchet limit can be accommodated within the existing method of the shakedown analysis $[12,16]$ where the linear elastic solution is augmented by the changing residual stress field $\rho_{i j}\left(t_{n}\right)$. The upper bound shakedown theorem is given by:

$$
\begin{gathered}
\int_{0 V}^{\Delta t} \int_{i j} \varepsilon_{i j}^{c} d V d t=\int_{0 V}^{\Delta t} \int_{i j}^{c} \sigma_{i j}^{c} d V d t \\
\hat{\sigma}_{i j}=\lambda \hat{\sigma}_{i j}^{\bar{F}}+\hat{\sigma}_{i j}^{\Delta}\left(x_{k}, t\right)+\rho_{i j}^{r}\left(x_{k}, t\right)
\end{gathered}
$$


where $\varepsilon_{i j}^{c}$ is kinematically admissible strain and $\sigma_{i j}^{c}$ denotes a state of associated stress with $\varepsilon_{i j}^{c}$ at yield. For the von Mises yield condition and the associated flow rule, we have

$$
\int_{0}^{\Delta t} \int_{V} \sigma_{i j}^{c} \varepsilon_{i j}^{c} d V d t=\int_{V} \sum_{n=1}^{N} \sigma_{y} \bar{\varepsilon}\left(\Delta \varepsilon_{i j}^{n}\right) d V
$$

where $\bar{\varepsilon}\left(\Delta \varepsilon_{i j}^{n}\right)=\sqrt{\frac{2}{3} \Delta \varepsilon_{i j}^{n} \Delta \varepsilon_{i j}^{n}}$ and $\sigma_{y}$ is the yield stress of material

Thus an upper bound on the ratchet limit multiplier can be obtained by

$$
\lambda=\frac{\int_{V} \sum_{n=1}^{N} \sigma_{y} \bar{\varepsilon}\left(\Delta \varepsilon_{i j}^{n}\right) d V-\int_{V} \sum_{n=1}^{N}\left(\hat{\sigma}_{i j}^{\Delta}\left(t_{n}\right)+\rho_{i j}\left(t_{n}\right)\right) \Delta \varepsilon_{i j}^{n} d V}{\int_{V} \hat{\sigma}_{i j}^{\bar{F}}\left(\sum_{n=1}^{N} \Delta \varepsilon_{i j}^{n}\right) d V}
$$

which gives the capacity of the body subjected to a predefined cyclic load history $\hat{\sigma}_{i j}^{\Delta}\left(t_{n}\right)$ to withstand an additional constant load $\hat{\sigma}_{i j}^{\bar{F}}$ before ratchetting takes place. On the basis of this formulation, the LMM produces a sequence of monotonically reducing upper bounds, which converges to the least upper bound ratchet limit for the chosen class of displacement fields. In the following sections, a centre cracked plate with circular holes is analysed in detail using the proposed method.

\section{Centre cracked plate with circular holes}

\subsection{Geometry}

The geometrical shape and the material properties of the centre cracked plate with symmetric drilled holes are as shown in Fig. 1 and Table1, respectively. The half-crack length $a$ is $500 \mathrm{~mm}$ and the ratios $\frac{W}{a}$ and $\frac{L}{a}$ are both 2 . The hole locations are referred to a co-ordinate system $X, Y$, the origin of which is located at the crack tip. $X_{0}$ and $Y_{0}$ are the coordinates of the hole's centre according to the coordinate system placed at the crack tip as shown in Fig.1. Calculations are made for hole diameters $D=40,50,100,125,150 \mathrm{~mm}$ with various symmetric drilled hole locations. The hole locations are defined by first drilling symmetric holes at various horizontal locations and keeping the vertical distance constant at $\frac{Y_{0}}{a}=0.3$. Once the optimum horizontal location has been found, this is then held constant and holes are drilled for various vertical locations.

\subsection{Loading}


The centre cracked plate is subjected to two different cyclic loading cases under plane strain condition. In the first case the plate is under cyclic tension loading with mean tension $\bar{\sigma}_{p}$, and in the second case a cyclic bending moment with reversed bending moment range $\Delta M$ and constant uniaxial tension $\bar{\sigma}_{p}$ is applied to the plate. The detailed cyclic loading histories are given in Fig.2, which show a cyclic loading history with two load extremes during each load cycle. For the cyclic tension case (Fig.2a), the two extremes of loading history can be formulated as $\sigma_{p}\left(t_{1}\right)=\bar{\sigma}_{p}+\Delta \sigma_{p} / 2$ and $\sigma_{p}\left(t_{2}\right)=\bar{\sigma}_{p}-\Delta \sigma_{p} / 2$, respectively, where $\bar{\sigma}_{p}$ represents the mean tensile loading and $\Delta \sigma_{p}$ represents the tension range. A similar loading history has also been modelled for the cyclic bending moment case (Fig.2b), by combining $\Delta M$, the reversed bending moment range, and $\bar{\sigma}_{p}$, the constant tensile loading. The reference mean tensile loading and the reference constant uniaxial tension, $\bar{\sigma}_{p o}$, with loading magnitude equal to $100 \mathrm{MPa}$ are used in both cases. A reference reversed bending moment range, $\Delta M_{0}=100 \mathrm{~N} . \mathrm{mm}$, is used for the cyclic bending moment case.

\subsection{FEM}

In the Finite Element Modelling (FEM) the sizes of the models are minimized by applying symmetry boundary conditions to quarter- and half-models, accordingly. The cyclic tensile loading case has two planes of symmetry, and for the cyclic bending moment case one plane of symmetry is used. Thus, only quarter- and half-model is required for the cyclic tension and cyclic bending moment cases, as shown in Fig. 3 and Fig.4, respectively. In both cases, along the symmetric axis, symmetric boundary conditions are imposed in the FEM. The analysis is performed using ABAQUS type CPE8R 8 node quadratic quadrilateral elements with reduced integration scheme.

\section{Comparison of the ratchet limit with limit load boundary}

In order to illustrate the effectiveness of the new ratchet limit method in cracked bodies, a centre cracked plate subjected to two different cyclic loading cases with a symmetrically located hole of diameter $D=100 \mathrm{~mm}$ is considered. The horizontal location, $X_{0}$, and the vertical location, $Y_{0}$, of the hole are kept at locations $\frac{X_{0}}{a}=-1, \frac{Y_{0}}{a}=0.3$, respectively.

\subsection{Cyclic tensile case}

In the case of the cyclic tensile loading, the converged values of upper bound ratchet limits obtained from the proposed LMM are shown in Fig.5 as an interaction diagram, composed of the limit for different ratios of varying tensile loading amplitude and the mean tension. The applied 
mean tension, $\bar{\sigma}_{P}$, in $\mathrm{X}$-axis and the tension amplitude $\Delta \sigma_{P} / 2$ in $\mathrm{Y}$-axis are normalized with respect to the reference tension $\bar{\sigma}_{p o}$. Fig.5 shows that the ratchet limit boundary coincides with limit load boundary, which is also calculated by the LMM. This means that any cyclic tensile load which exceeds the ratchet limit will also exceed the limit load and cause plastic collapse in the first cycle (i.e. there will be no ratchetting or incremental plastic collapse which normally occurs due to the excessive cyclic loads). The coincidence of the ratchet limit and limit load boundaries is due to the fact that for both the ratchet limit analysis with the cyclic tensile load history, and the limit load analysis with static tensile load, the maximum tensile load during the cycle is dominant and leads to the same plastic collapse in both cases. The accuracy of the limit load boundary by the LMM has been verified by ABAQUS RIKS analysis, which provides the same limit load boundary as that calculated by the LMM. For the verification of ratchet limit boundary calculated by the LMM the cyclic load point $\mathrm{C}\left(\Delta \sigma_{p}=2 \bar{\sigma}_{p 0}, \bar{\sigma}_{p}=\bar{\sigma}_{p 0}\right)$, which is just below the calculated ratchet limit boundary (Fig.5), is chosen for the step-by-step analysis in ABAQUS. The plastic strain history at the crack tip for the cyclic loading $\mathrm{C}$ is shown in Fig.6, where the $\mathrm{Y}$-axis represents the normalized maximum equivalent plastic strain for the cyclic load point $C$ (Fig.5) with respect to maximum equivalent plastic strain at the crack tip for a centre cracked plate without holes under the action of the reversed tension $\left(\Delta \sigma_{p}=2 \bar{\sigma}_{p 0}, \bar{\sigma}_{p}=0\right)$. As expected, Fig.6 shows a reverse plasticity mechanism under the cyclic load case $\mathrm{C}$, where the maximum equivalent plastic strain calculated by the stepby-step analysis ceases to increase at about 3 load cycles and settles into a closed loop for the remaining cycles. This observation confirms the predicted ratchet limit curve.

\subsection{Cyclic bending moment case}

The same procedure is also applied to the cyclic bending moment case, and the interaction diagram is shown in Fig.7, where the applied constant pressure in X-axis is normalized with respect to the reference uniaxial tension $\bar{\sigma}_{p o}$, while the cyclic bending moment in Y-axis is normalized using the reference cyclic bending moment $\Delta M_{0}$. Unlike in the cyclic tensile loading case, the ratchet limit and the limit load curves do not coincide, which means that an increase in the loads beyond the ratchet limit will not automatically cause plastic collapse. Any combination of loads which lies between these two boundaries will result in ratchetting. The accuracy of the limit load boundary obtained by the LMM has been verified by ABAQUS RIKS analysis, which provides the same limit load boundary as that calculated by the LMM. For the verification of ratchet limit boundary calculated by the LMM the cyclic load points $\mathrm{D}\left(\Delta M=1.6 \Delta M_{0}, \bar{\sigma}_{p}=\bar{\sigma}_{p 0}\right)$, and $\mathrm{E}$ ( $\Delta M=1.6 \Delta M_{0}, \bar{\sigma}_{p}=1.1 \bar{\sigma}_{p 0}$ ), which are just below and above the calculated ratchet limit boundary

(Fig.7), respectively, are chosen for the step-by-step analysis in ABAQUS. The plastic strain history 
at the crack tip for the cyclic loading D and E are shown in Fig.8, where the Y-axis represents the normalized maximum equivalent plastic strain for the cyclic load points D and E (Fig.7) with respect to maximum equivalent plastic strain at the crack tip for a centre cracked plate without holes under the action of the reversed bending moment $\left(\Delta M=1.6 \Delta M_{0}, \bar{\sigma}_{p}=0\right)$. The calculated maximum equivalent plastic strain for the load case $D$ settles to a stable cycle after about 5 load cycles showing a reverse plasticity mechanism, and the load case $E$ shows a strong ratcheting mechanism, with the maximum equivalent plastic strain increasing at every cycle. Thus, the results in Fig.8 obtained by ABAQUS step-by-step analysis confirm the accuracy of the predicted ratchet limits by the LMM for the cyclic bending moment case.

\section{Results}

The effect of the hole location and the hole size on ratchet limit and maximum plastic strain range for the centre cracked plate are analyzed in this study. Firstly, symmetric holes are drilled at various horizontal locations keeping the vertical distance constant at $\frac{Y_{0}}{a}=0.3$. Once the optimum point is reached subsequent analyses are performed with varying vertical coordinates and fixed horizontal location.

\subsection{The effect of the hole location and size on ratchet limit in horizontal direction}

The ratchet limit interaction curve for a centre cracked plate with drilled holes of diameter $\mathrm{D}=100 \mathrm{~mm}$ at different horizontal locations (keeping the vertical distance constant at $\frac{Y_{0}}{a}=0.3$ ) is shown in Fig.9 for both cyclic loading cases. It is observed from Fig. 9 that at different levels of cyclic tension and bending moment the ratchet limit boundary will always show the trend to sharply decrease as the holes move toward the ligament side (from $\frac{X_{0}}{a}=0$ to $\frac{X_{0}}{a}=0.3$ ) and it will remain almost constant when the holes move from the crack tip to the centre of the plate (from $\frac{X_{0}}{a}=0$ to $\left.\frac{X_{0}}{a}=-1\right)$

Since the above trend of results is valid for any cyclic loading point, in the coming discussions we only consider the results of the ratchet limit and maximum plastic strain range calculated at the cyclic loading point $\left(\Delta \sigma_{p} / 2\right) / \bar{\sigma}_{p o}=1$ for the cyclic tensile loading case and $(\Delta M / 2) / \Delta M_{0}=1$ for the cyclic bending moment case. The variation of the normalized ratchet limit multiplier $\lambda_{R} / \lambda_{R 0}$ for various hole diameters and locations is shown in Fig.10a and Fig. 10b, where the ratchet limit 
multiplier $\lambda_{R}$, in the $\mathrm{Y}$ direction, is normalized with respect to that of a centre cracked plate without holes, $\lambda_{R 0}$.

It is observed in Fig.10a that from point $\frac{X_{0}}{a}=-1\left(\frac{Y_{0}}{a}=0.3\right)$, to point $\frac{X_{0}}{a}=-0.2\left(\frac{Y_{0}}{a}=0.3\right)$, all the ratchet limit multipliers for various hole sizes are nearly having the same value as that in the case without holes, therefore the ratchet limit is unaffected by the presence of the hole within the range of diameters considered here. From location $\frac{X_{0}}{a}=-0.2$ to location $\frac{X_{0}}{a}=-0.1$ the normalized ratchet limit multiplier decreases slightly. Beyond hole location $\frac{X_{0}}{a}=-0.1$ the normalized ratchet limit multiplier falls sharply. This decrease in the figure is shown up to point $\frac{X_{0}}{a}=0.3$. Between hole location $\frac{X_{0}}{a}=-0.2$ and $\frac{X_{0}}{a}=-0.1$ in Fig.10a, it is shown that the ratchet limit multiplier is decreasing less than $0.1 \%$ for diameters $40-50 \mathrm{~mm}$, and less than $2 \%$ for diameters $100-150 \mathrm{~mm}$ compared to the case of a centre cracked plate without holes. From location $\frac{X_{0}}{a}=-0.1$ to location $\frac{X_{0}}{a}=0.3$ the drop of the ratchet limit multiplier increases proportionally with the increasing hole diameter.

Fig. 10b shows the variation of the normalized ratchet limit multiplier with moving holes in horizontal direction for the cyclic bending moment case. It is observed from Fig.10b that the holes start to show the effect on ratchet limit multiplier at location $\frac{X_{0}}{a}=-0.1$ while for the cyclic tensile loading case this effect is observed to start at point $\frac{X_{0}}{a}=-0.2$. From location $\frac{X_{0}}{a}=-0.1$ to location $\frac{X_{0}}{a}=0.3$, more significant decrease in normalized ratchet limit multiplier is identified at the same hole location when compared with the cyclic tensile loading case.

\subsection{Effect of hole location and size on plastic strain range in horizontal direction}

The variation of calculated normalized maximum plastic strain range $\Delta \varepsilon_{p} / \Delta \varepsilon_{p 0}$ for various hole diameters is shown in Fig.11, where the maximum plastic strain range $\Delta \varepsilon_{p}$ in the $\mathrm{Y}$ direction is normalized with respect to that of a centre cracked plate without holes, $\Delta \varepsilon_{p 0}$. 
It is observed in Fig.11a that from point $\frac{X_{0}}{a}=-1\left(\frac{Y_{0}}{a}=0.3\right)$, to point $\frac{X_{0}}{a}=-0.3\left(\frac{Y_{0}}{a}=0.3\right)$, the maximum plastic strain range is unaffected by the presence of the holes regardless of the hole size. From location $\frac{X_{0}}{a}=-0.3$ to location $\frac{X_{0}}{a}=-0.1$ the maximum plastic strain range falls sharply and reaches its minimum at location $\frac{X_{0}}{a}=-0.1$. Beyond hole location $\frac{X_{0}}{a}=-0.1$ there is a drastic increase in the maximum plastic strain range and this increase reaches its maximum at point $\frac{X_{0}}{a}=0.1$. Between hole location $\frac{X_{0}}{a}=-0.3$ and $\frac{X_{0}}{a}=-0.1$ in Fig.11a it is observed that greater reductions in the maximum plastic strain range are given by larger hole diameters. It is also observed in the figure that at location $\frac{X_{0}}{a}=-0.1$ a hole diameter of 40 or $50 \mathrm{~mm}$ has little effect on the maximum plastic strain range (causing differences of $1 \%$ and $3 \%$ respectively). Larger hole diameters at this location have a more significant effect, with differences between $30 \%$ and $50 \%$ in the maximum plastic strain range observed for diameters between $100 \mathrm{~mm}$ and $150 \mathrm{~mm}$. Beyond point $\frac{X_{0}}{a}=-0.1$ (as the hole is moving from the crack tip toward the ligament side), the bigger the hole diameter is, the greater the increase of the maximum plastic strain range will be.

Fig. 11b shows the trend of the maximum plastic strain range for a plate with a hole moving in horizontal direction while the vertical direction is kept constant for the cyclic bending moment case. Compared to the cyclic tensile loading case, the moving holes show a similar effect on the maximum plastic strain range however the hole size has a more profound effect. As per the cyclic tensile case, diameters of $40-50 \mathrm{~mm}$ show little effect on the maximum plastic strain range (1\% and $2 \%$, respectively). Larger hole diameters, however, show a greater effect, with reductions of 40$72 \%$ in the maximum plastic strain range resulting from hole diameters of $100-150 \mathrm{~mm}$.

It can be seen from Figs. 10 and 11 that the largest decrease in maximum plastic strain range is given by a hole at a distance of $\frac{X_{0}}{a}=-0.1$ from the crack tip. This hole location of $\frac{X_{0}}{a}=-0.1$ is considered to be the horizontal optimum location.

\subsection{Effect of hole location and size on ratchet limit in vertical direction}

Keeping the horizontal optimum location $\left(\frac{X_{0}}{a}=-0.1\right)$ constant, holes are drilled at various vertical locations. The variation of ratchet limit multiplier in different vertical positions for both cyclic loading cases is shown in Fig.12a-12b, where the ratchet limit multiplier $\lambda_{R}$ in the $\mathrm{Y}$ direction is normalized with respect to that of a centre cracked plate without holes, $\lambda_{R 0}$. It can be seen from 
these figures that at $\frac{X_{0}}{a}=-0.1$, the vertical height of the holes have no effect on the ratchet limit multiplier.

\subsection{The effect of the hole location and size on plastic strain range in vertical direction}

The variation of maximum plastic strain range in different vertical positions for both cyclic loading cases is shown in Fig.13a-13b, where the maximum plastic strain range $\Delta \varepsilon_{p}$ in the $\mathrm{Y}$ direction is normalized with respect to the one in the case of a centre cracked plate without holes, $\Delta \varepsilon_{p 0}$. It is seen in Fig.13a that as the holes move in the positive Y direction (Fig.1), the maximum plastic strain range increases for the cyclic tensile loading case. From hole location $\frac{Y_{0}}{a}=0.3$ to $\frac{Y_{0}}{a}=0.8$ it can be observed that larger hole sizes will give smaller maximum plastic strain range. The normalized value of maximum plastic strain range asymptotically approaches to unity irrespective of the hole size when the holes move away from the crack and after location $\frac{Y_{0}}{a}=0.7$ the difference between hole sizes becomes negligible. At location $\frac{Y_{0}}{a}=0.3\left(\frac{X_{0}}{a}=-0.1\right)$, the decrease in maximum plastic strain range is maximum, $1 \%$ and $3 \%$ for diameters $40-50 \mathrm{~mm}$ and $30 \%$ to $50 \%$ for diameters $100 \mathrm{~mm}$ to $150 \mathrm{~mm}$. The similar but more significant behavior is observed in the cyclic bending moment case. At location $\frac{Y_{0}}{a}=0.3\left(\frac{X_{0}}{a}=-0.1\right)$, the decrease in maximum plastic strain range is maximum, $1 \%$ and $2 \%$ for diameters $40-50 \mathrm{~mm}$ and $40 \%$ to $72 \%$ for diameters $100 \mathrm{~mm}$ to $150 \mathrm{~mm}$.

\subsection{The optimum hole location and size}

It can be concluded from above discussions that the optimum location, where the decrease in maximum plastic range is maximum and the reduction in ratchet limit is minimum, is located at point $\frac{X_{0}}{a}=-0.1, \frac{Y_{0}}{a}=0.3$. It is also observed that from the hole diameters considered here, a $150 \mathrm{~mm}$ diameter hole is shown to be the most beneficial. At this optimum hole location and size, the maximum decrease in plastic strain range is $50 \%$ and the corresponding ratchet limit is $2 \%$, for the cyclic tensile case. For cyclic bending, this hole diameter and location gives a $72 \%$ reduction in the maximum plastic strain range and does not reduce the ratchet limit.

\section{Discussions}


The above results could be explained by Fig.14 and Fig.15, which show the failure pattern at the limit state for both cyclic loading cases with various horizontal locations by keeping vertical distance constant at $\frac{Y_{0}}{a}=0.3$. Both cyclic loading cases have similar failure patterns at the ratchet limit state for the same horizontal location. Fig.14a and Fig.15a show that without the holes, the failure pattern appears with a 45 degree angle linking from the crack tip to the edge of the plate. When the hole is drilled at the horizontal locations $\frac{X_{0}}{a}=0.2, \frac{X_{0}}{a}=0.1$ and $\frac{X_{0}}{a}=0$ (Fig.14b-Fig.14d and Fig.15b-Fig.15d) which are within the failure area, the failure pattern at the ratchet limit state becomes discontinuous due to the presence of the holes, which weakens the plate's strength. These are the reasons why the ratchet limit boundary and normalized ratchet limit multiplier are decreasing in these locations (Fig.9 and Fig.10). At hole location $\frac{X_{0}}{a}=-0.1$ (Fig.14e and Fig.15e), which is just outside the failure area, the stress concentration and stress field produced by the holes interact with that of the crack which reduces the maximum strain range, and also causes a slight reduction on the ratchet limit. When the holes are placed far from the failure area (beyond $\frac{X_{0}}{a}=-0.2$ in Fig.14f and Fig.15f), it causes no effect on the failure pattern. That is why the ratchet limit multiplier has the same value as that in the case of a plate without holes and so does the maximum plastic strain range.

\section{Conclusion}

In the present study, the effect of circular holes on maximum plastic strain range and the ratchet limit has been studied using the proposed Linear Matching Method. The new LMM has been verified by step-by-step analysis and RIKS analyses in ABAQUS, showing that it gives very accurate ratchet limits for a plate with a centre cracked and symmetrically drilled holes under complex cyclic loading histories. Parametric studies involve holes with different diameters drilled at different locations. The optimum location where the maximum plastic strain range decreases the most with minimum effect on the ratchet limit is located at a distance $10 \%$ of the semi-cracked length from crack tip opposite the ligament for both the cyclic tensile loading and cyclic bending moment cases. And it is also observed that the location is independent of hole sizes. The most significant decrease in maximum plastic strain range is observed as $50 \%$ with $2 \%$ reduction in the ratchet limit, for the hole size $\mathrm{D}=150 \mathrm{~mm}$ at the optimum location $\frac{X_{0}}{a}=-0.1, \frac{Y_{0}}{a}=0.3$ in the cyclic tension case. For cyclic bending, this hole diameter and location gives a $72 \%$ reduction in the plastic strain range and does not reduce the ratchet limit. 


\section{Acknowledgements}

The authors gratefully acknowledge the support of the Engineering and Physical Sciences Research Council (EP/G038880/1) of the United Kingdom, and the University of Strathclyde during the course of this work.

\section{References}

1. R. Grief, J.L. Sanders Jr. The effect of a stringer on the stress in a cracked sheet. J. Appl. Mech 1965; 32: 59 \pm 66 .

2. F. Erdogan, K. Arin. A sandwich plate with a part through and a debonding crack. J. Eng. Fract.

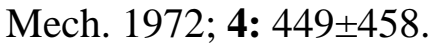

3. S.B. Thomas, M.J. Mhaiskar, Raju Sethuraman. Stress intensity factors for circular hole and inclusion using finite element alternating method. Theoretical and Applied Fracture Mechanics. 2000; 33:73-81.

4. Melan E. Theorie Statisch Unbestimmter Systeme aus Ideal-Plastischem Bastoff. Sitzungsberichte der Akademie der Wissenschaft,Wien, Abtiia. 1936; 145:195-218.

5. Staat M., Heitzer M. LISA a European Project for FEM-based Limit and Shakedown Analysis. Nuclear Engineering and Design. 2001; 206: 151-166.

6. Vu, D.K, Yan, A.M, Nguyen-Dang, H. A primal-dual algorithm for shakedown analysis of structures. Comput. Methods Appl. Mech. Eng. 2004; 193: 4663-4674.

7. Seshadri, R. Inelastic Evaluation of Mechanical and Structural components Using the Generalized Local Stress Strain Method of Analysis. Nucl. Eng. Des. 1995; 153: 287-303.

8. Mackenzie, D, Boyle, J.T, Hamilton, R. The elastic compensation method for limit and shakedown analysis: a review. Trans IMechE, Journal of Strain Analysis for Engineering Design. 2000; 35:171-188.

9. Ponter, A.R.S, \& Chen, H.F. A minimum theorem for cyclic load in excess of shakedown, with application to the evaluation of a ratchet limit. European Journal of Mechanics - A/Solids. 2001; 20:539-553.

10. Chen HF, Ponter ARS. A method for the evaluation of a ratchet limit and the amplitude of plastic strain for bodies subjected to cyclic loading. European Journal of Mechanics - A/Solids. 2001; 20: 555-571.

11. ABAQUS. User's manual. Version 6.7. 2007; 
12. Chen, H.F. Lower and Upper Bound Shakedown Analysis of structures With TemperatureDependent Yield Stress. Journal of Pressure Vessel Technology. 2010; 132:1-8

13. Chen, H.F. A Direct Method on the Evaluation of Ratchet Limit. Journal of Pressure Vessel Technology. 2010; 132: 041202

14. Chen, H.F. \& Ponter, A.R.S. Linear Matching Method on the evaluation of plastic and creep behaviours for bodies subjected to cyclic thermal and mechanical loading. International Journal for Numerical Methods in Engineering. 2006; 68:13-32.

15. Broek. D. A Similitude Criterion for Fatigue Crack Growth Modeling. Fracture Mechanics Sixteenth Symposium, ASTM STP 868. M. F. Kanaien and A.T. Hopper. Eds. American Society for Testing and Material, Philadelphia. 1985; 347-360

16. Chen, H.F., Ponter ARS. Shakedown and limit analyses for 3-D structures using the Linear Matching Method. International Journal of Pressure Vessels and Piping. 2001;78:443-451. 


\section{Table Captions}

Table 1 Material properties of the steel

Table 1. Material properties of the steel

\begin{tabular}{llll}
\hline $\begin{array}{l}\text { Young's modulus } \\
E(\mathrm{GPa})\end{array}$ & $\begin{array}{l}\text { Poisson's } \\
\text { ratio } v\end{array}$ & $\begin{array}{l}\text { Coefficient of thermal } \\
\text { expansion } \alpha\left({ }^{\circ} C^{-1}\right)\end{array}$ & $\begin{array}{l}\text { Yield stress } \\
\sigma_{y}(\mathrm{MPa})\end{array}$ \\
\hline 200 & 0.32 & $1.11 \times 10^{-5}$ & 360 \\
\hline
\end{tabular}




\section{Figure Captions}

Figure 1. Centre cracked plate with symmetric holes subjected to cyclic tensile loading and cyclic bending moment

Figure 2. (a) The cyclic tensile loading history with mean tension $\bar{\sigma}_{p}$ and tension range $\Delta \sigma_{p}$ (b) The cyclic bending moment history with reversed bending moment range $\Delta M$ and constant tension $\bar{\sigma}_{p}$

Figure 3. (a) Quarter symmetry model for cyclic tensile loading case (b) Finite element model

Figure 4. (a) Half symmetry model for cyclic bending moment case (b) Finite element model

Figure 5. Ratchet limit interaction curve for the cyclic tensile loading case with hole location at $\frac{X}{a}=-0.1, \quad \frac{Y}{a}=0.3(\mathrm{D}=100 \mathrm{~mm})$

Figure 6. ABAQUS verification of the ratchet limit for the cyclic tensile loading case using detailed step by step analysis

Figure 7. Ratchet limit interaction curve for the cyclic bending moment case with hole location at $\frac{X}{a}=-0.1, \quad \frac{Y}{a}=0.3(\mathrm{D}=100 \mathrm{~mm})$

Figure 8. ABAQUS verification of the ratchet limit for the cyclic bending moment case using detailed step by step analysis

Figure 9. Ratchet limit interaction curve with varying horizontal hole location and fixed vertical location at $Y / a=0.3(\mathrm{D}=100 \mathrm{~m})$ : (a) cyclic tensile loading case and (b) cyclic bending moment case

Figure 10. Variation of normalized ratchet limit multiplier with varying horizontal hole location at the fixed vertical location $\left(Y / a=0.3\right.$ ): (a) for cyclic tensile loading case $\left(\Delta \sigma_{p} / 2\right) / \bar{\sigma}_{p o}=1$ (Fig.9a) and (b) for cyclic bending moment case $(\Delta M / 2) / \Delta M_{0}=1$ (Fig.9b)

Figure 11. Variation of normalized maximum plastic strain range with varying horizontal hole location at the fixed vertical location $(Y / a=0.3)$ : (a) for cyclic tensile loading case $\left(\Delta \sigma_{p} / 2\right) / \bar{\sigma}_{p o}=1$ (Fig.9a) and (b) for cyclic bending moment case $(\Delta M / 2) / \Delta M_{0}=1$ (Fig.9b)

Figure 12. Variation of normalized ratchet limit multiplier with varying vertical hole location at fixed horizontal location ( $X / a=-0.1$ ): (a) for cyclic tensile loading case $\left(\Delta \sigma_{p} / 2\right) / \bar{\sigma}_{p o}=1$ (Fig.9a) and (b) for cyclic bending moment case $(\Delta M / 2) / \Delta M_{0}=1$ (Fig.9b) 
Figure 13. Variation of normalized maximum plastic strain range with varying vertical hole location at fixed horizontal location ( $X / a=-0.1$ ): (a) for cyclic tensile loading case $\left(\Delta \sigma_{p} / 2\right) / \bar{\sigma}_{p o}=1$ (Fig.9a) and (b) for cyclic bending moment case $(\Delta M / 2) / \Delta M_{0}=1$ (Fig.9b)

Figure 14. Fracture pattern at the limit state for cyclic tensile loading case with different horizontal hole location (fixed vertical location $Y / a=0.3$ ) at: (a) without hole; (b) $X / a=0.2$;(c) $X / a=0.1$;(d) $X / a=0$;(e) $X / a=-0.1$ and (f) $X / a=-0.2$

Figure 15. Fracture pattern at the limit state for cyclic bending moment case with different horizontal hole location (fixed vertical location $Y / a=0.3$ ) at: (a) without hole; (b) $X / a=0.2$;(c) $X / a=0.1$;(d) $X / a=0$;(e) $X / a=-0.1$ and (f) $X / a=-0.2$ 


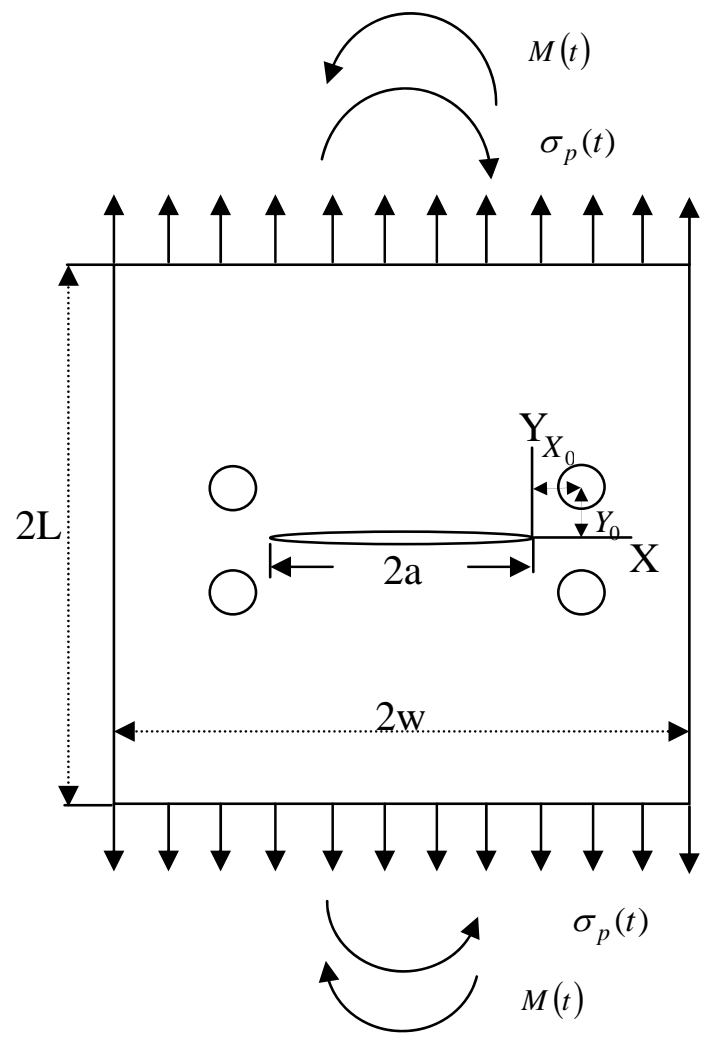

Figure 1. Centre cracked plate with symmetric holes subjected to cyclic tensile loading and cyclic bending moment 


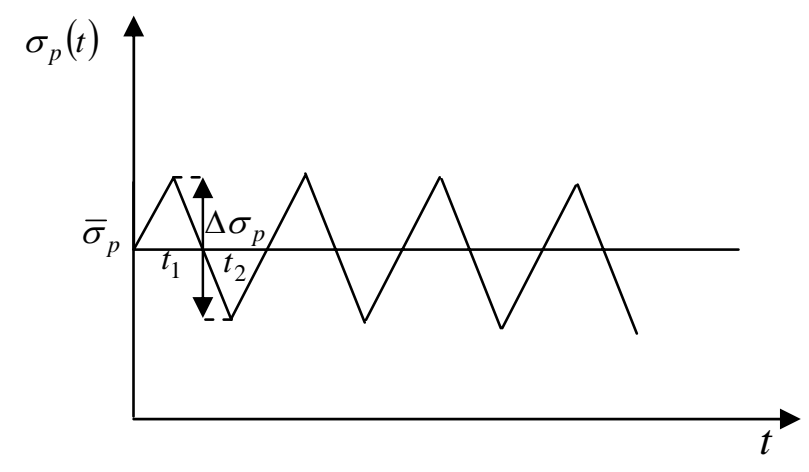

(a)
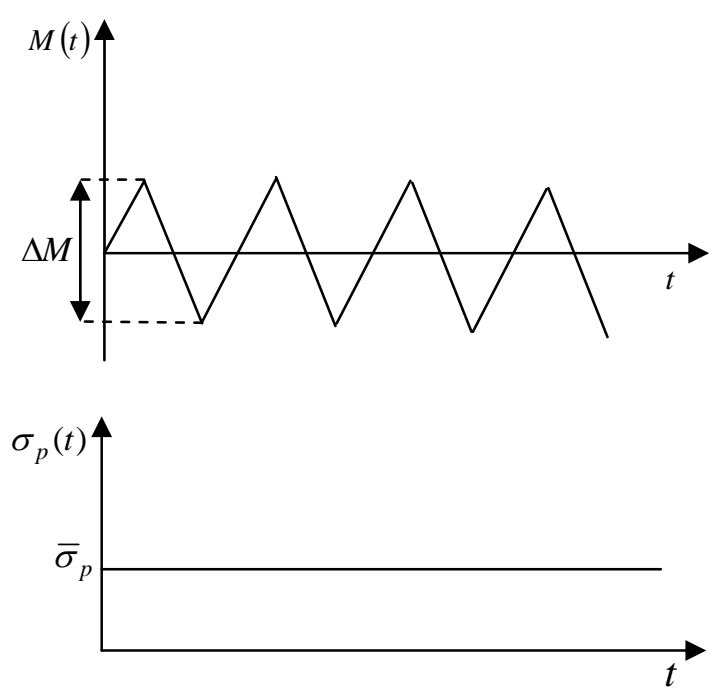

(b)

Figure 2. (a) The cyclic tensile loading history with mean tension $\bar{\sigma}_{p}$ and tension range $\Delta \sigma_{p}$ (b) The cyclic bending moment history with reversed bending moment range $\Delta M$ and constant tension $\bar{\sigma}_{p}$ 

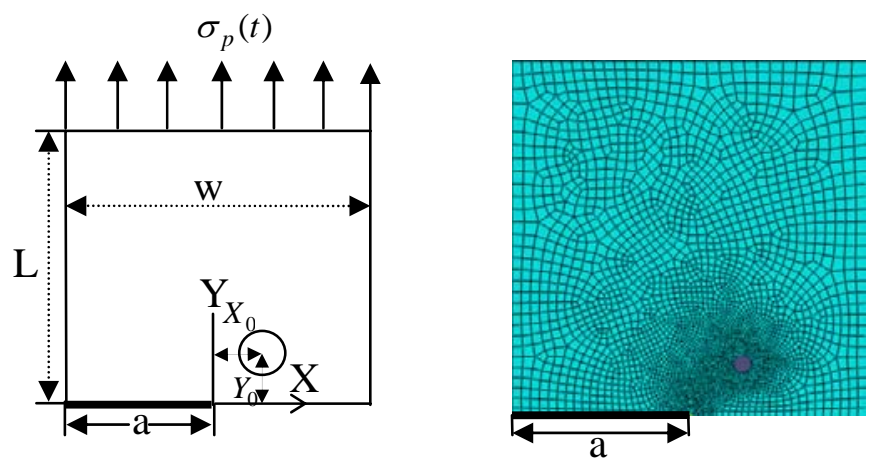

Figure 3. (a) Quarter symmetry model for cyclic tensile loading case (b) Finite element model 


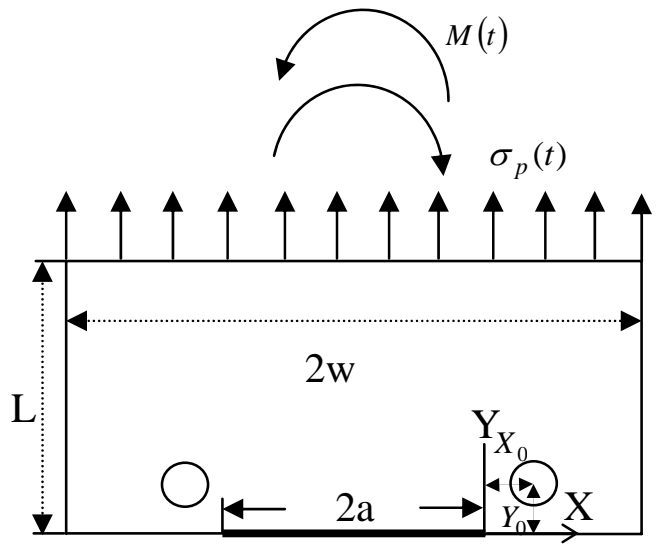

(a)

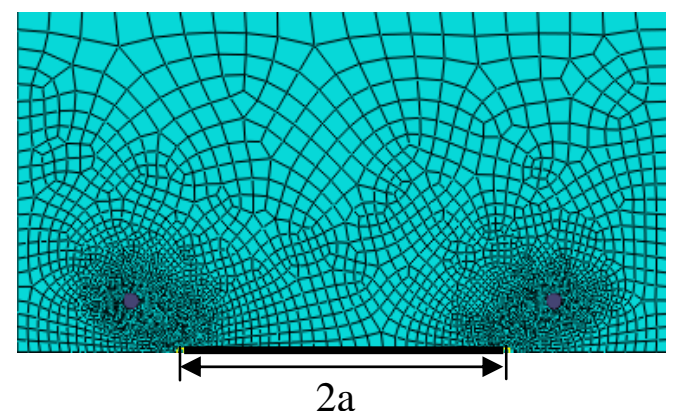

(b)

Figure 4. (a) Half symmetry model for cyclic bending moment case (b) Finite element model 


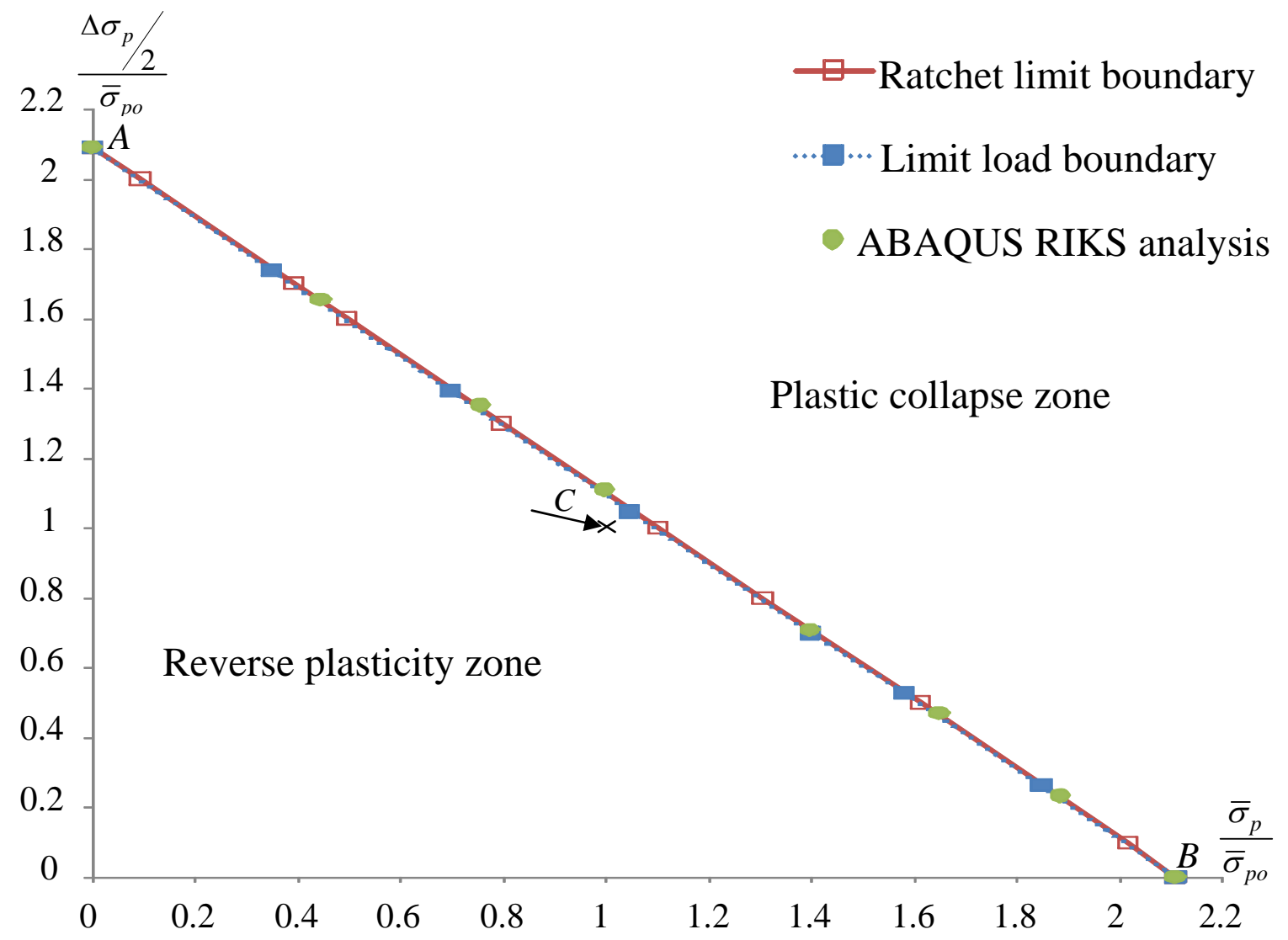

Figure 5. Ratchet limit interaction curve for the cyclic tensile loading case with hole location at $\frac{X}{a}=-0.1, \frac{Y}{a}=0.3 \quad(\mathbf{D}=\mathbf{1 0 0} \mathbf{m m})$ 


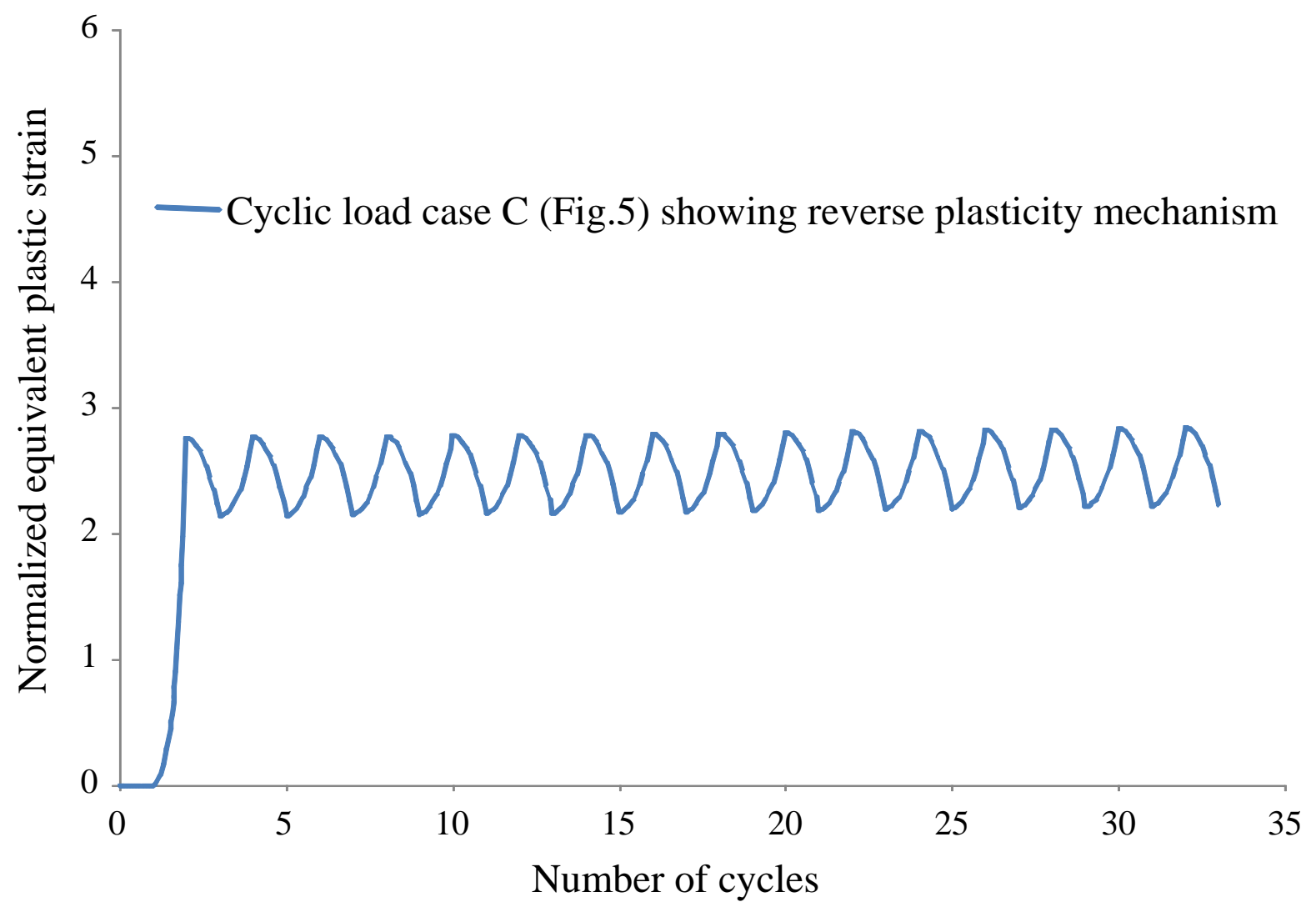

Figure 6. ABAQUS verification of the ratchet limit for the cyclic tensile loading case using detailed step by step analysis 


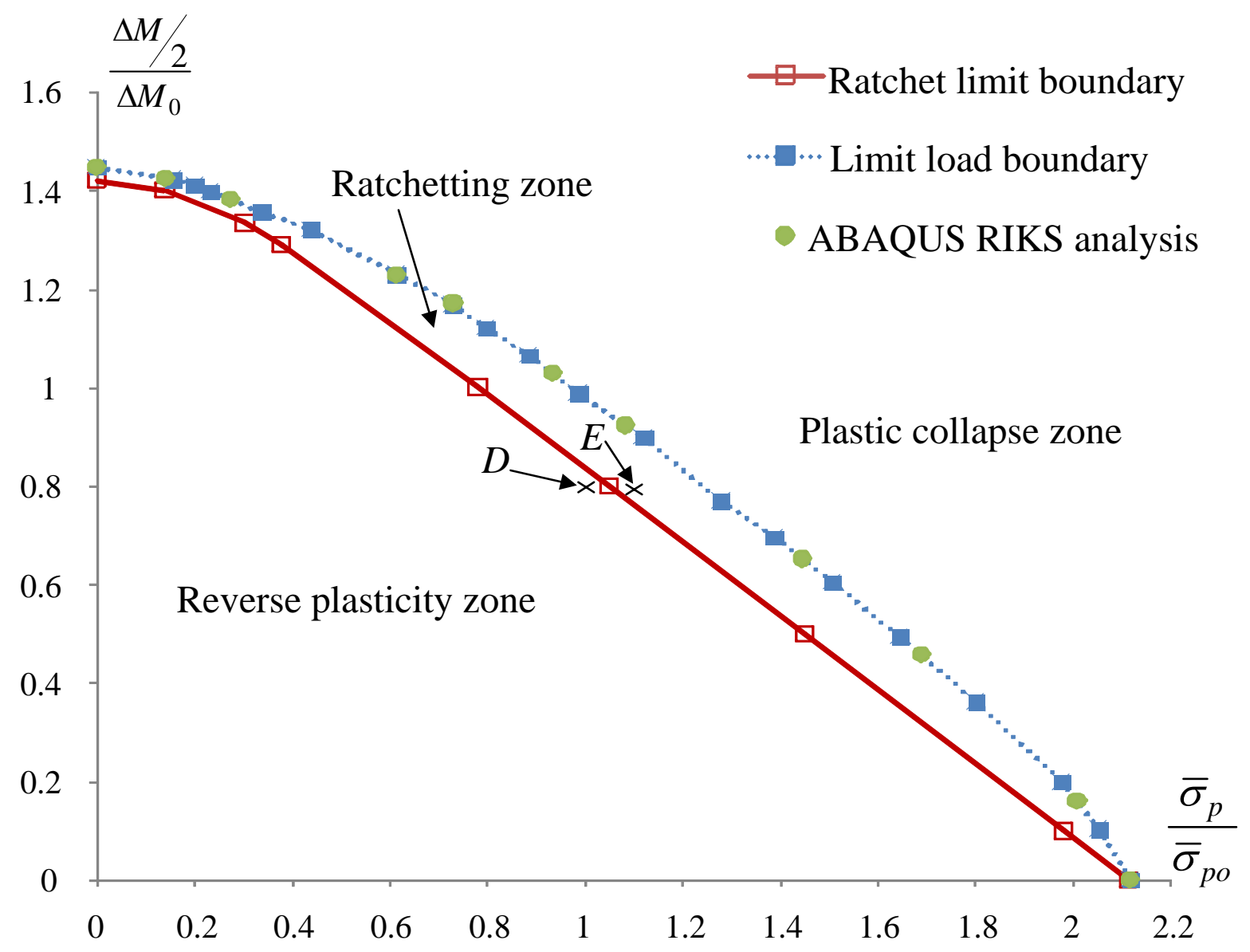

Figure 7. Ratchet limit interaction curve for the cyclic bending moment case with hole location at $\frac{X}{a}=-0.1, \frac{Y}{a}=0.3 \quad(\mathbf{D}=\mathbf{1 0 0} \mathbf{m m})$ 


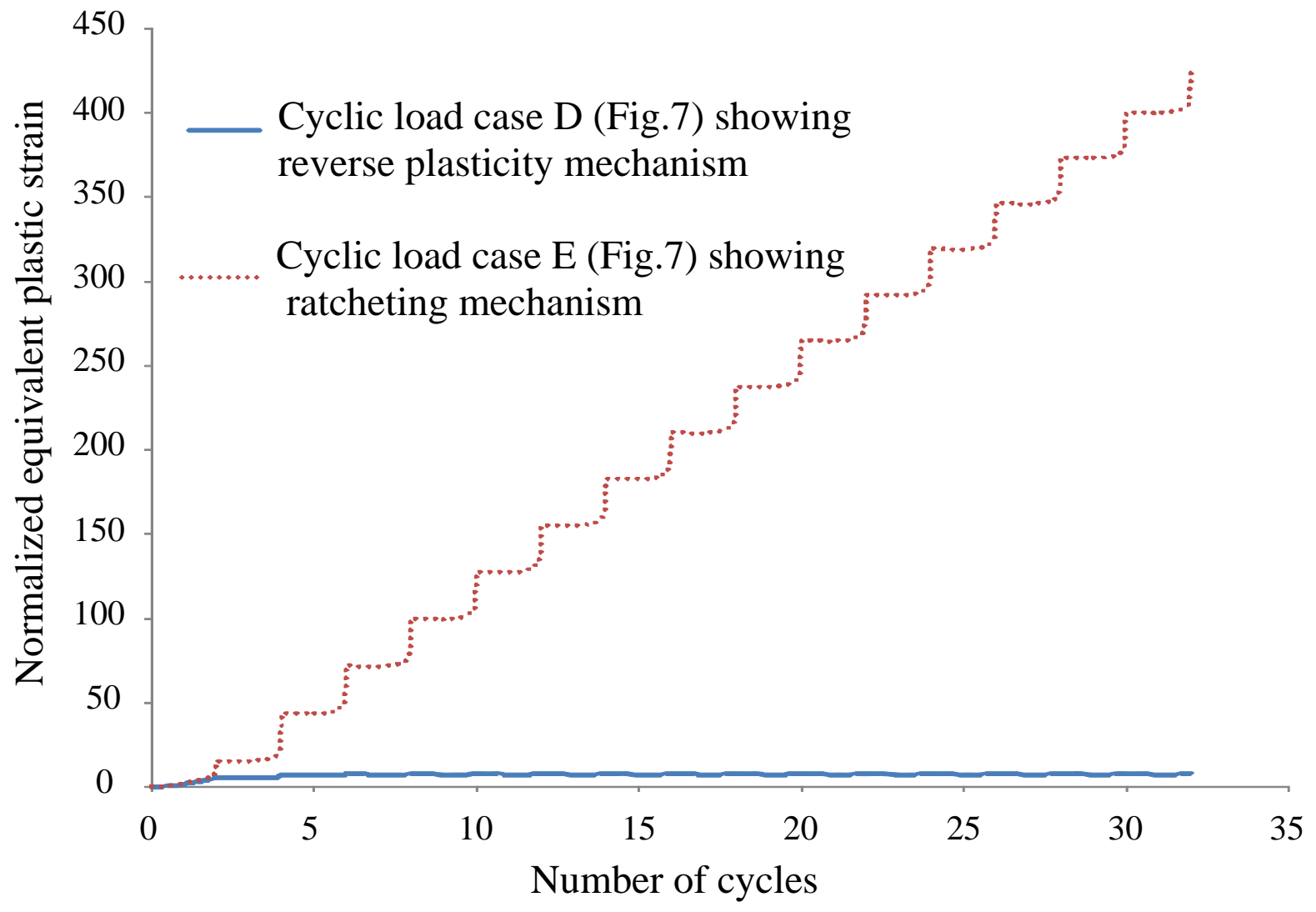

Figure 8. ABAQUS verification of the ratchet limit for the cyclic bending moment case using detailed step by step analysis 


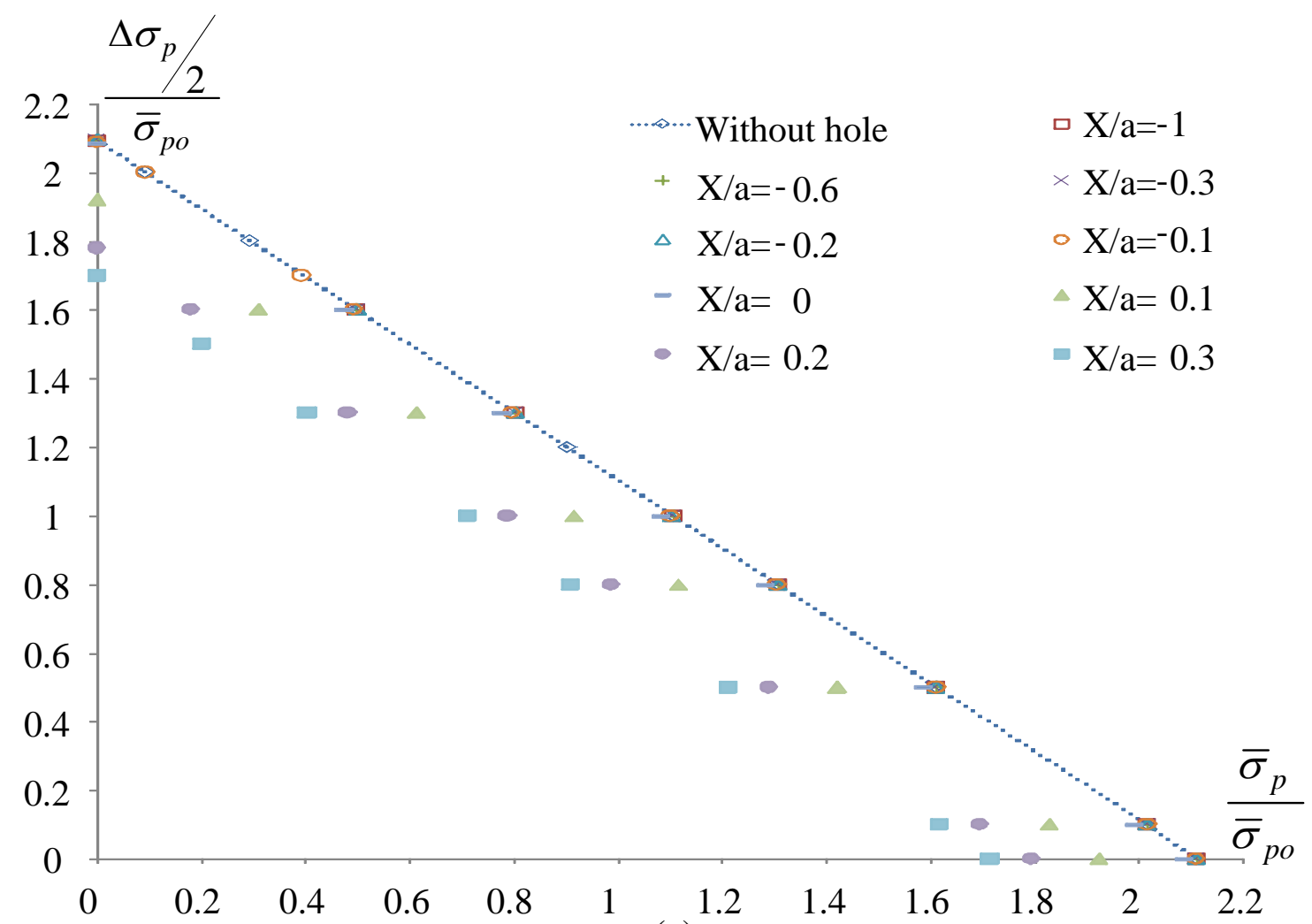

(a)

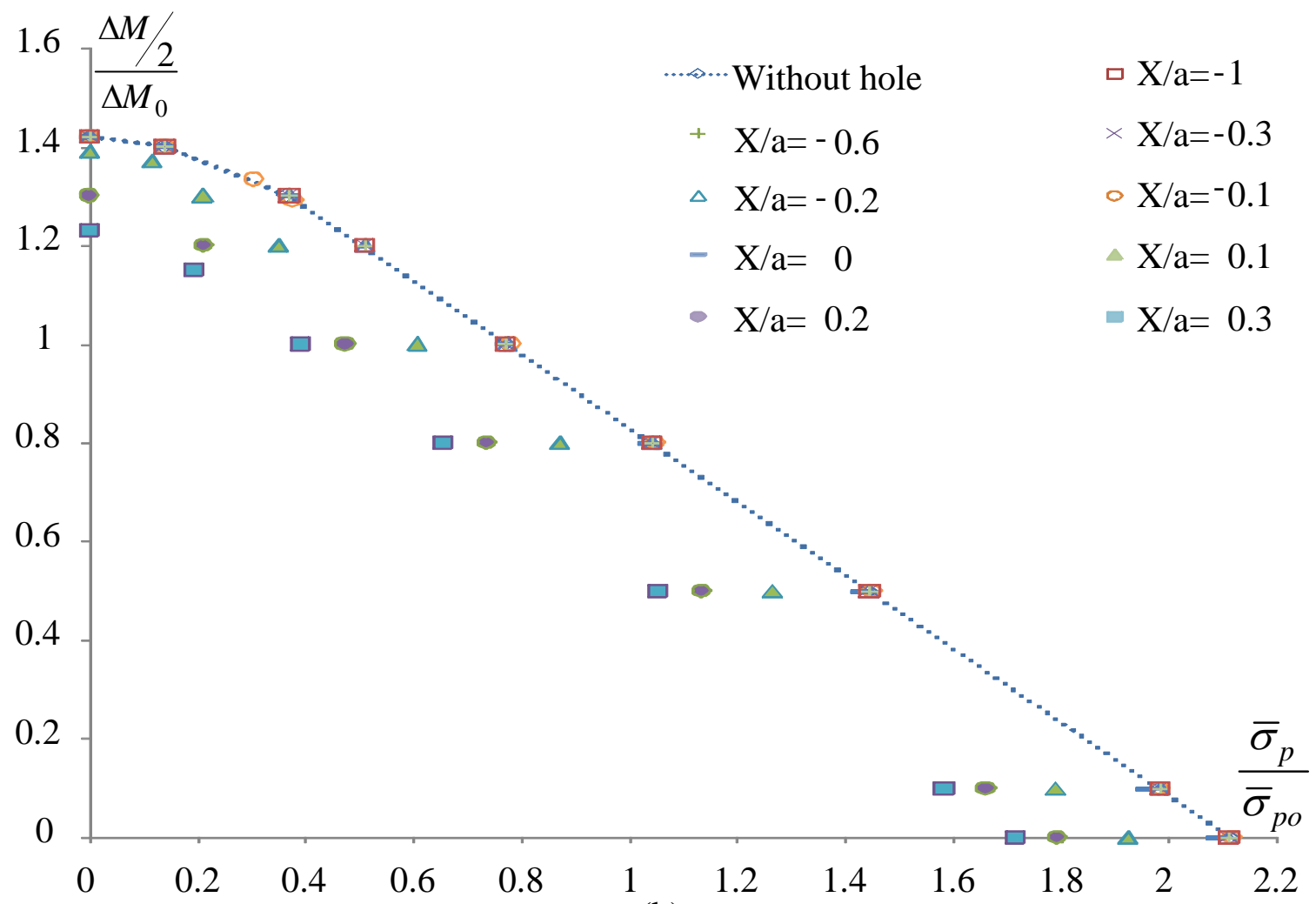

(b)

Figure 9. Ratchet limit interaction curve with varying horizontal hole location and fixed vertical location at $Y / a=0.3(\mathbf{D}=\mathbf{1 0 0} \mathbf{m})$ : (a) cyclic tensile loading case and (b) cyclic bending moment case 


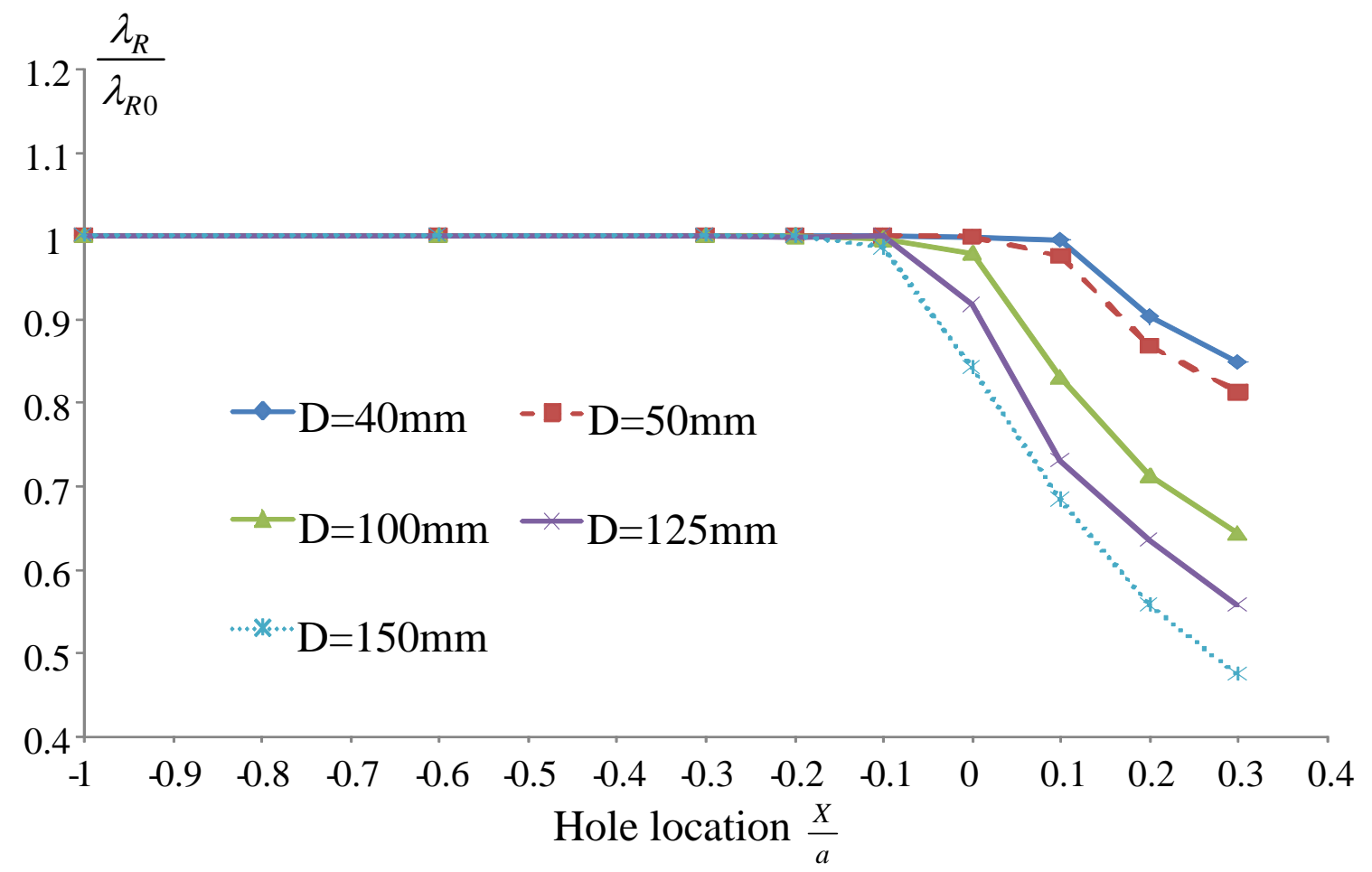

(a)

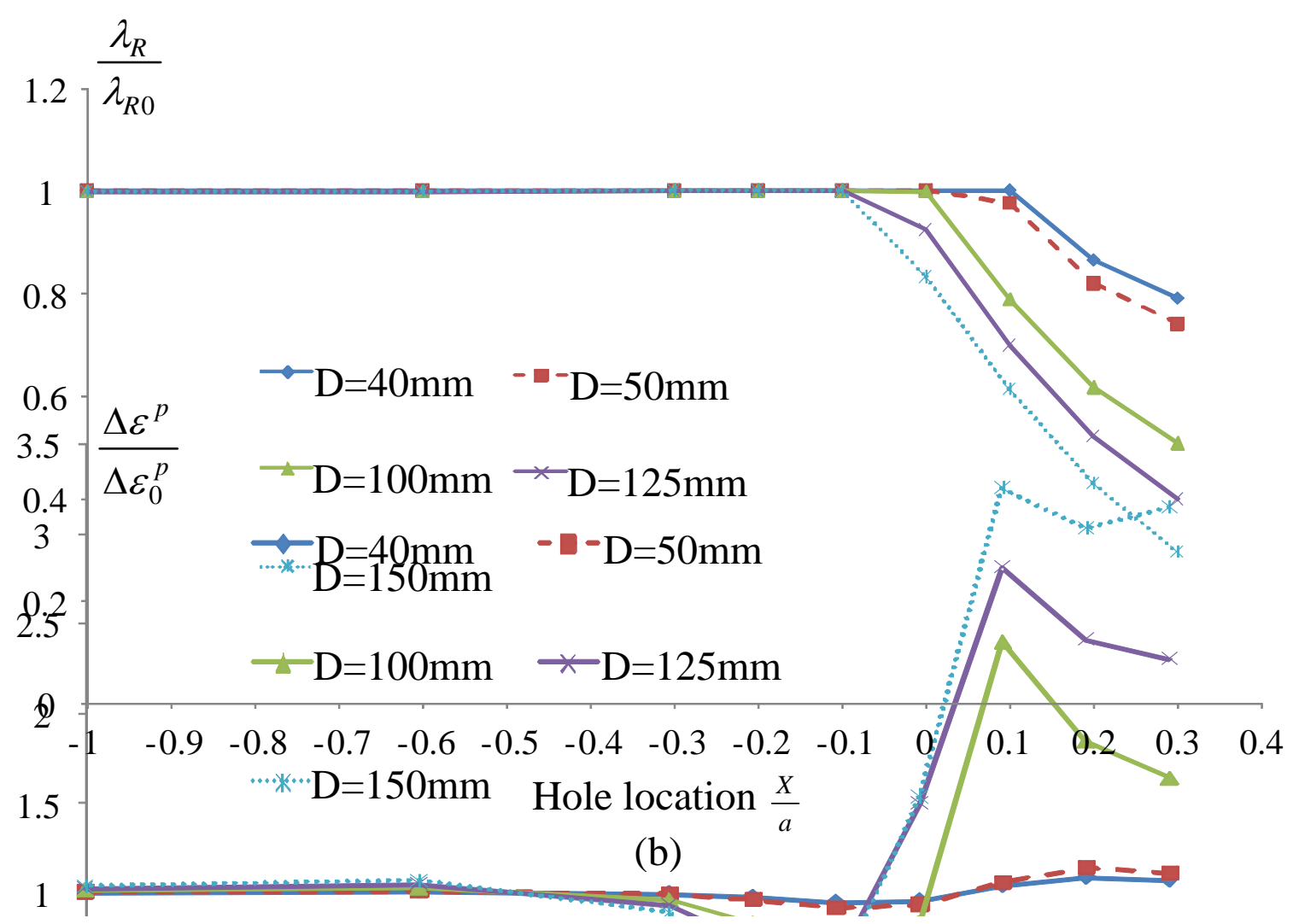

Figure 10. Variation of normalized ratchet limit multiplier with varying horizontal hole location at the fixed vertical location $(Y / a=0.3$ ): (a) for cyclic tensile loading case $\left(\Delta \sigma_{p} / 2\right) / \bar{\sigma}_{p o}=1$ (Fig.9a) and (b) for cyclic bending moment case $(\Delta M / 2) / \Delta M_{0}=1$ (Fig.9b)

Hole location $\frac{X}{a}$

(a)

$3 \frac{\Delta \varepsilon^{p}}{\Delta \varepsilon_{0}^{p}}$ 
Figure 11. Variation of normalized maximum plastic strain range with varying horizontal hole location at the fixed vertical location ( $Y / a=0.3$ ): (a) for cyclic tensile loading case $\left(\Delta \sigma_{p} / 2\right) / \bar{\sigma}_{p o}=1$ (Fig.9a) and (b) for cyclic bending moment case $(\Delta M / 2) / \Delta M_{0}=1$ (Fig.9b)

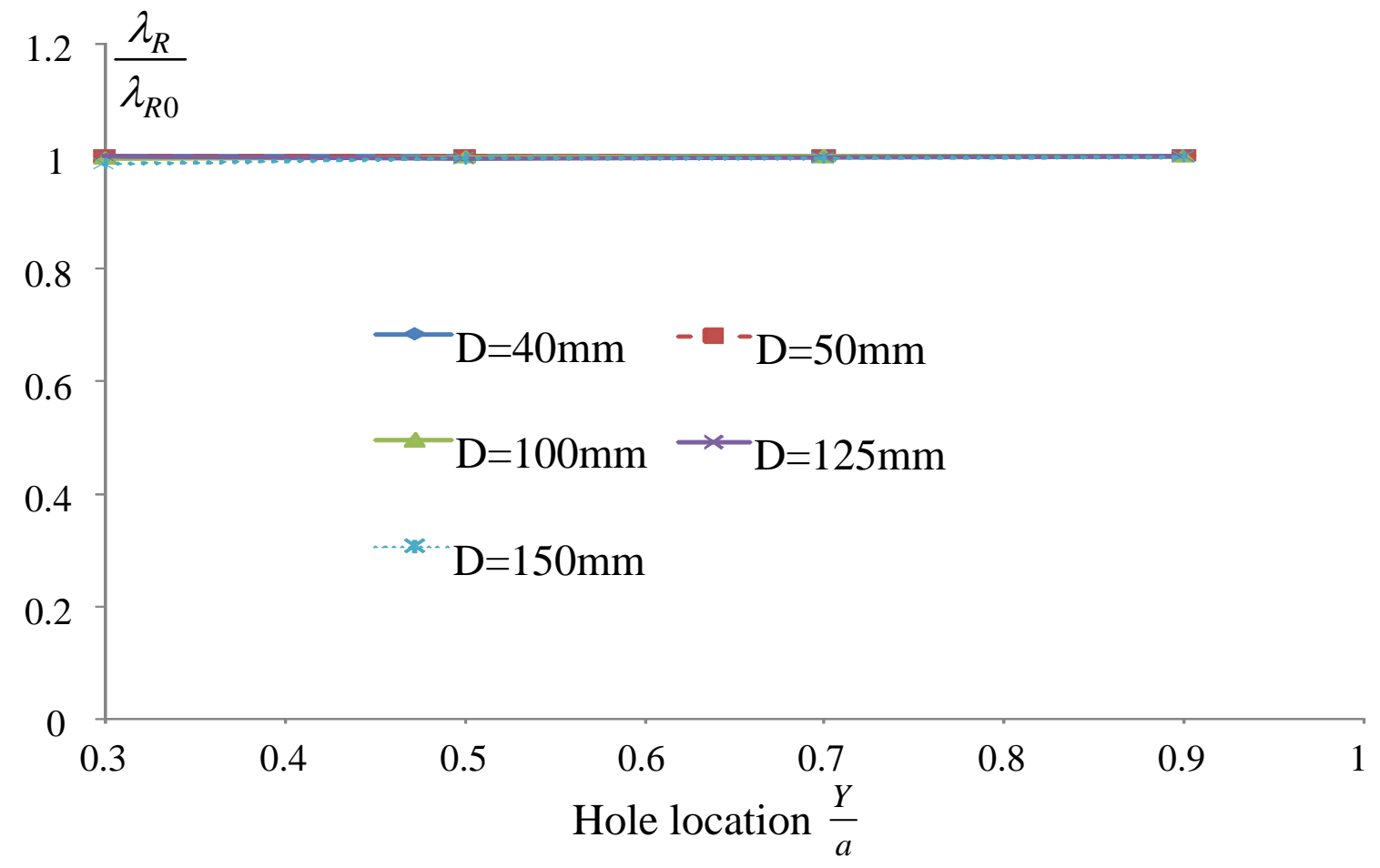

(a)

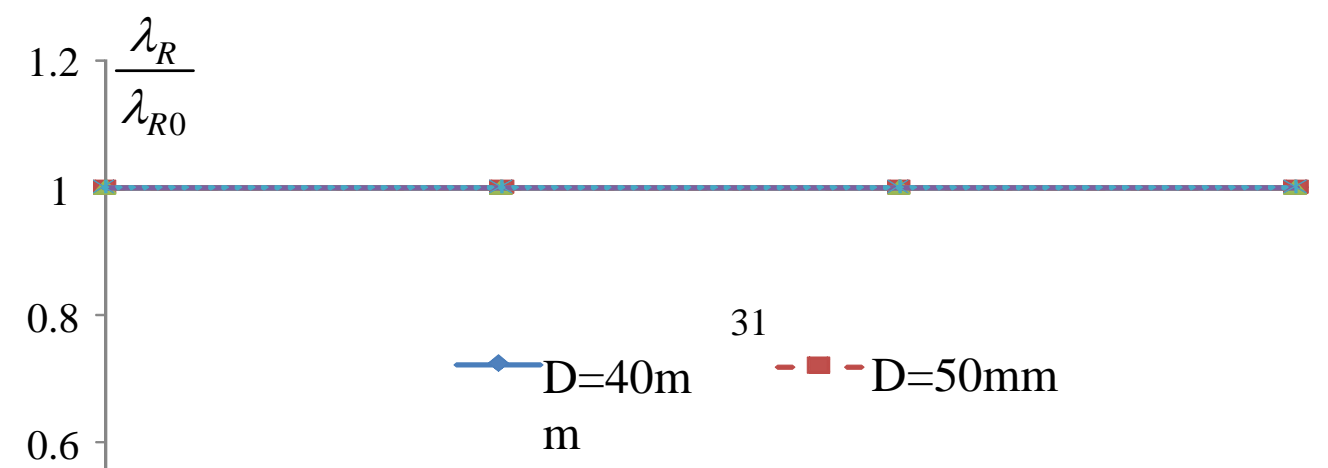




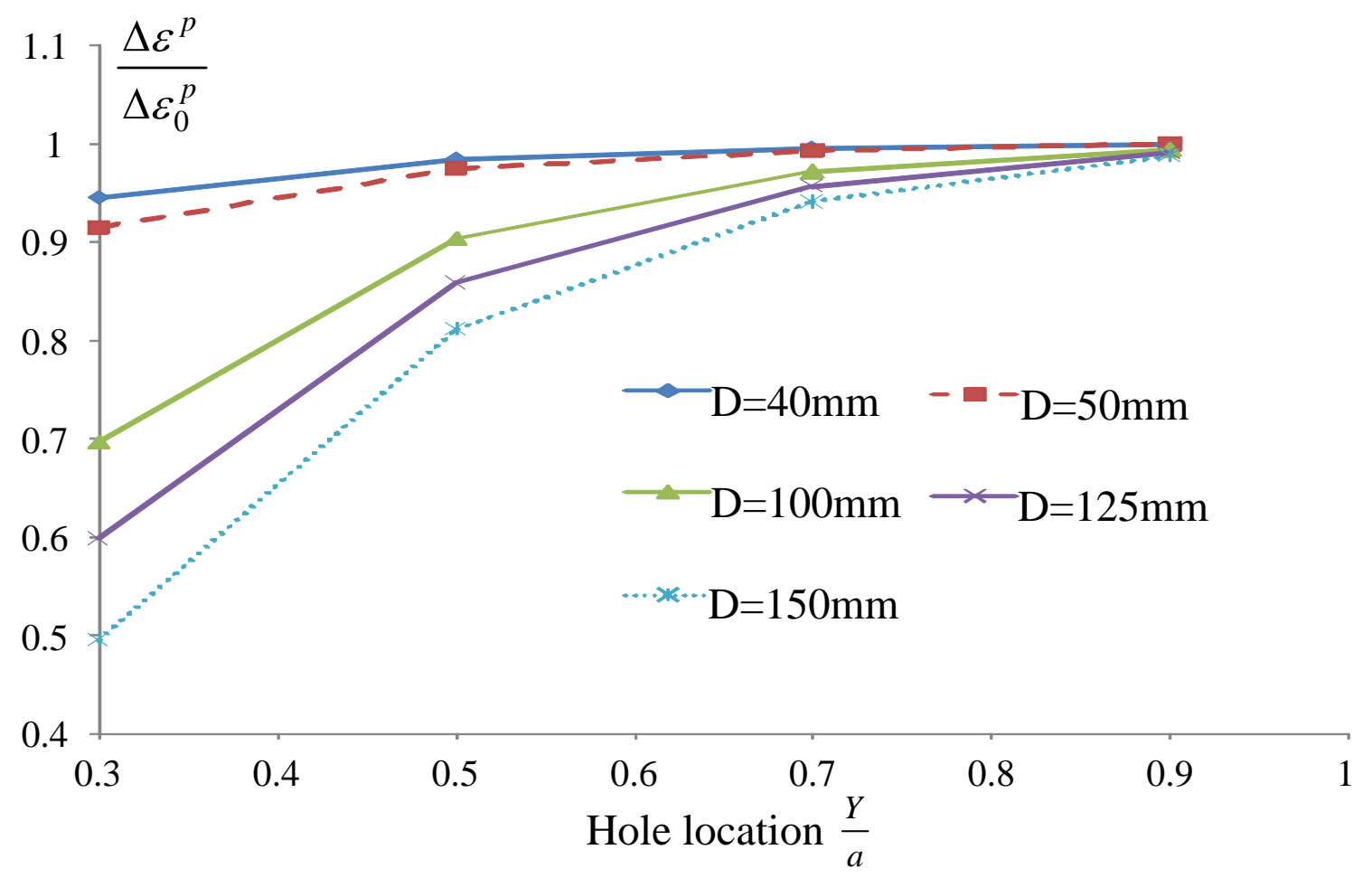

(a)

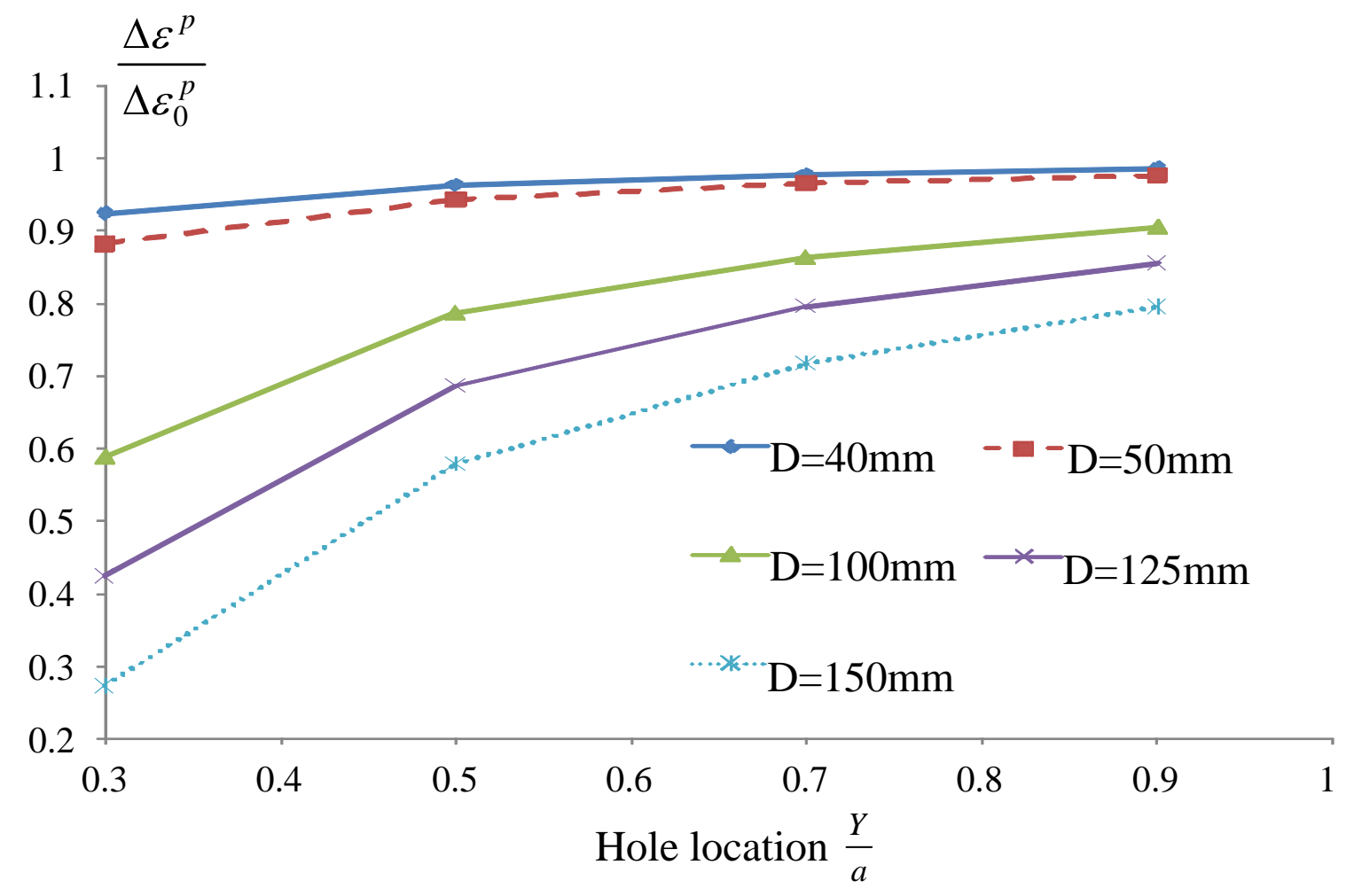

(b)

Figure 13. Variation of normalized maximum plastic strain range with varying vertical hole location at fixed horizontal location ( $X / a=-0.1$ ): (a) for cyclic tensile loading case $\left(\Delta \sigma_{p} / 2\right) / \bar{\sigma}_{p o}=1$ (Fig.9a) and (b) for cyclic bending moment case $(\Delta M / 2) / \Delta M_{0}=1$ (Fig.9b) 


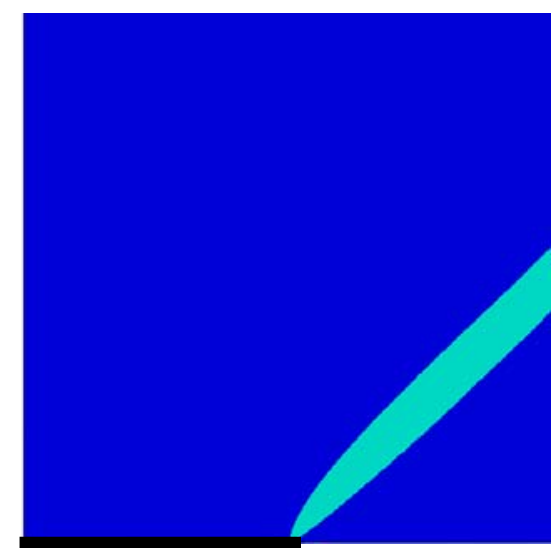

(a)

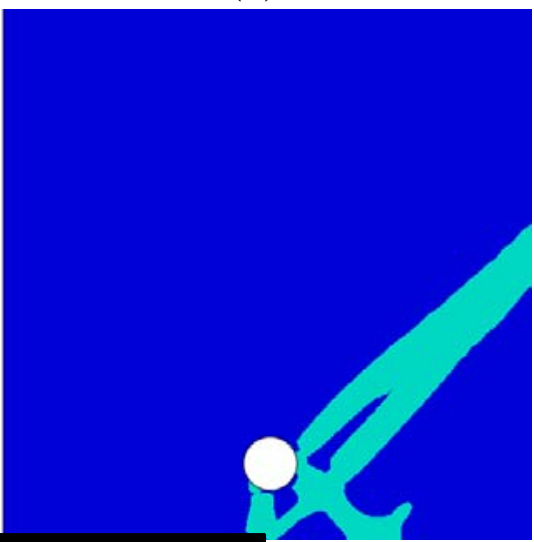

(d)

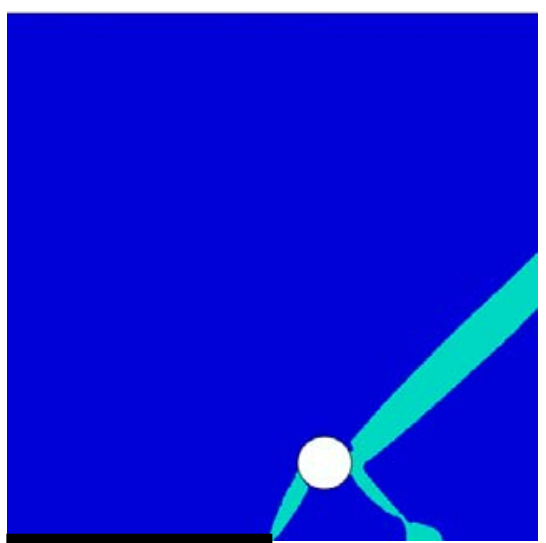

(b)

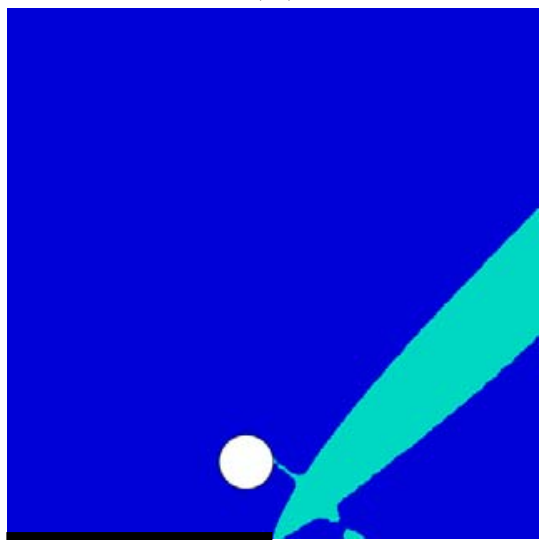

(e)

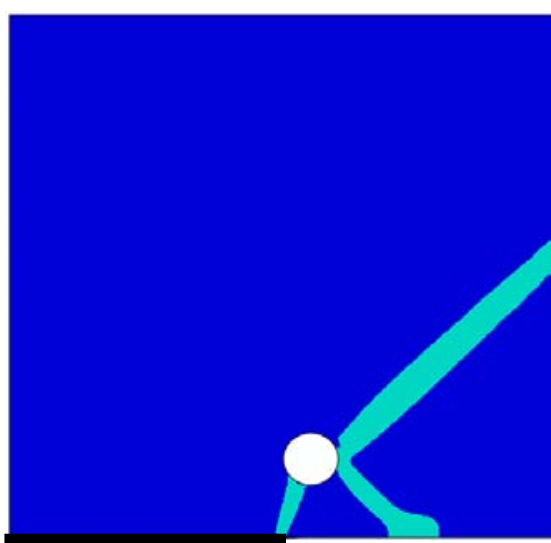

(c)

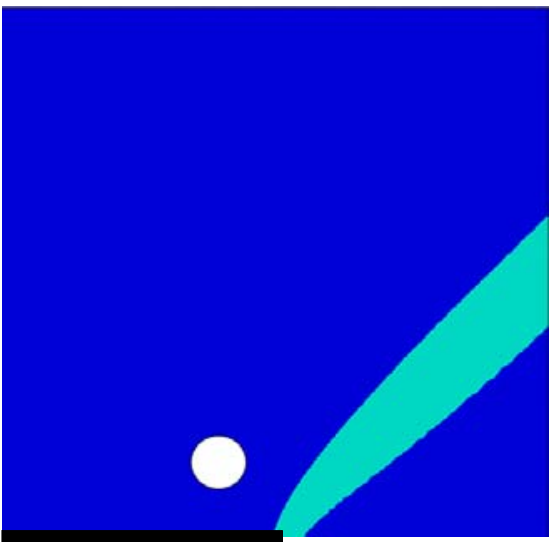

(f)

Figure 14. Fracture pattern at the limit state for cyclic tensile loading case with different horizontal hole location (fixed vertical location $Y / a=0.3$ ) at: (a) without hole; (b) $X / a=0.2$;(c) $X / a=0.1$;(d) $X / a=0$;(e) $X / a=-0.1$ and (f) $X / a=-0.2$ 


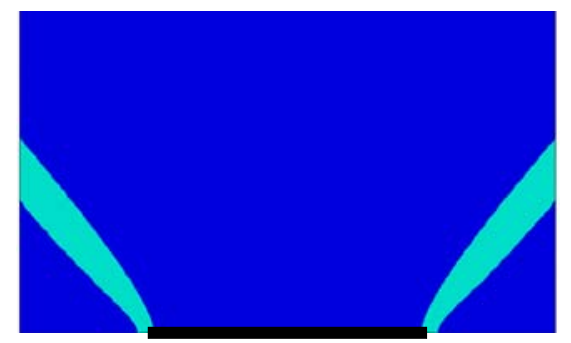

(a)

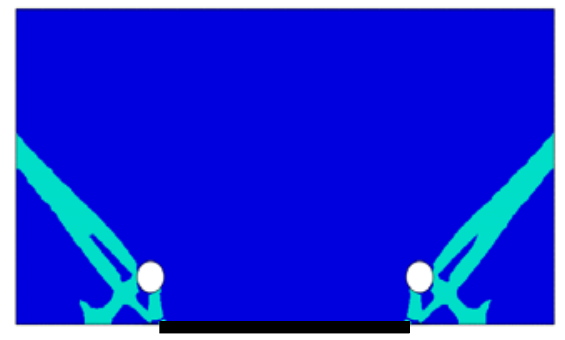

(d)

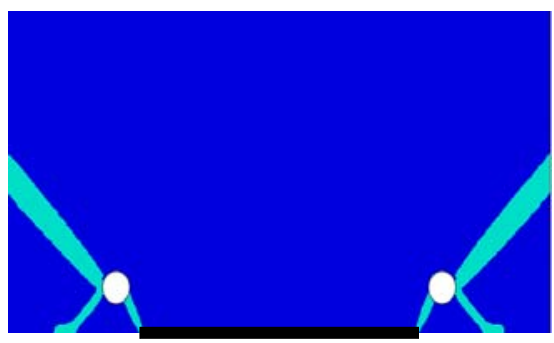

(b)

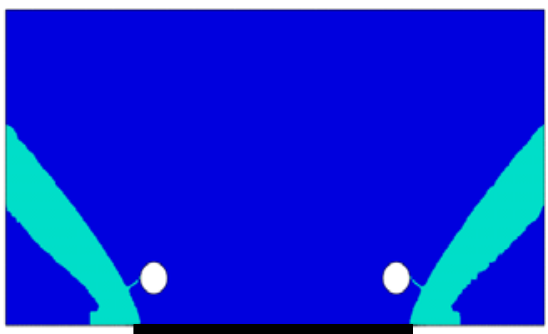

(e)

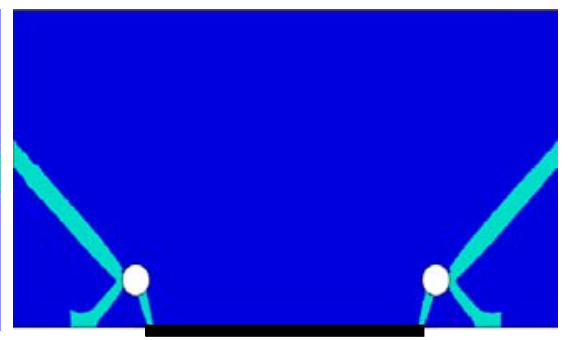

(c)

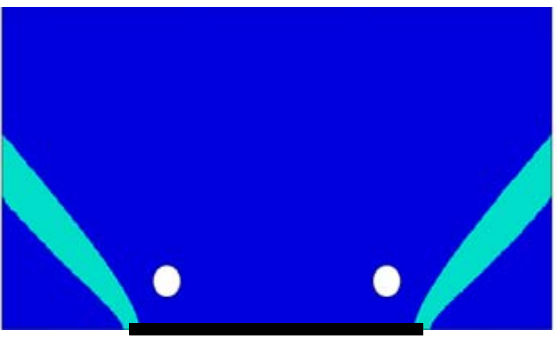

(f)

Figure 15. Fracture pattern at the limit state for cyclic bending moment case with different horizontal hole location (fixed vertical location $Y / a=0.3$ ) at: (a) without hole; (b) $X / a=0.2$;(c) $X / a=0.1 ;$ (d) $X / a=0$;(e) $X / a=-0.1$ and (f) $X / a=-0.2$ 